\title{
A Fully Portable High Performance Minimal Storage Hybrid Format Cholesky Algorithm
}

\author{
Bjarne S. Andersen \\ UNI•C Danish IT Center for Education and Research \\ and \\ John A. Gunnels and Fred G. Gustavson \\ IBM T.J. Watson Research Center \\ and \\ John K. Reid \\ Atlas Centre, Rutherford Appleton Laboratory \\ and \\ Jerzy Waśniewski \\ Technical University of Denmark
}

\begin{abstract}
We consider the efficient implementation of the Cholesky solution of symmetric positive-definite dense linear systems of equations using packed storage. We take the same starting point as that of LINPACK and LAPACK, with the upper (or lower) triangular part of the matrix being stored by columns. Following LINPACK and LAPACK, we overwrite the given matrix by its Cholesky factor. We consider the use of a hybrid format in which blocks of the matrices are held contiguously and compare this to the present LAPACK code. Code based on this format has the storage advantages of the present code, but substantially outperforms it. Furthermore, it compares favourably to using conventional full format (LAPACK) and using the recursive format of Andersen, Gustavson, and Waśniewski.
\end{abstract}

Categories and Subject Descriptors: G.1.3 [Numerical Analysis]: Numerical Linear Algebra Linear Systems (symmetric and Hermitian); G.4 [Mathematics of Computing]: Mathematical Software

General Terms: Algorithms, BLAS, Performance.

Additional Key Words and Phrases: real symmetric matrices, complex Hermitian matrices, positive definite matrices, Cholesky factorization and solution, recursive algorithms, novel packed matrix data structures, linear systems of equatons.

\section{INTRODUCTION}

It was apparent by the late 1980s, when the Level-3 BLAS [Dongarra et al. 1990] were designed, that blocking would be needed to obtain high performance on com-

Authors' addresses: B. A. Andersen, UNI•C, Building 304, DTU Lyngby, Denmark DK-2800; J.A. Gunnels and F.G. Gustavson, IBM T.J. Watson Research Center, Yorktown Heights NY, 10598, USA; J. K. Reid, Atlas Centre, Rutherford Appleton Laboratory, Didcot, Oxon OX11 OQX, UK; J. Waśniewski, Department of Informatics and Mathematical Modeling, Building 305, DTU Lyngby, Denmark DK-2800. 
puters with multi-level memory systems (see, for example, Calahan [1986], IBM [1986], and Gallivan, Jalby, Meier, and Sameh [1987]). This approach is now firmly established. More recently, it has become recognized that the most significant Level-3 BLAS is _GEMM, which performs the computation

$$
C=\alpha o p(A) o p(B)+\beta C
$$

where $A, B$, and $C$ are matrices held in conventional full storage, $o p(A)$ is $A$ or $A^{T}, o p(B)$ is $B$ or $B^{T}$, and $\alpha$ and $\beta$ are scalars. Agarwal, Gustavson, and Zubair [1994] and, later, Whaley, Petitet, and Dongarra [2000], point out that this can be performed particularly efficiently when $o p(A)=A^{T}$ and $o p(B)=B$ on any cache-based machine if $A$ is small enough to remain in the level-1 cache while the columns of $B$ and $C$ are read into the cache and the changed columns of $C$ are written out. The cache does not need to be big enough to hold all three arrays; rather, it needs to be big enough to hold $A$ and a few columns of $B$ and $C$. This mode of working is called 'streaming'. Note that it requires $B$ and $C$ to be held by columns. Multiplying by $A^{T}$ rather than $A$ facilitates the calculation of inner products.

If $A$ is too big for the cache or $B$ or $C$ is not held by columns, the benefits of streaming may be obtained by making copies in contiguous memory of blocks of a suitable size. We will refer to this as 'data copy'. For very large matrices, this will be an insignificant overhead, but for medium-sized blocks arising within a bigger calculation such as Cholesky factorization, the data-copy overhead may be significant.

On a computer with a single level of cache, it is easy enough to choose a suitable block size. With more than one level of cache, nested blocking is desirable and Gustavson [1997], Chatterjee, Jain, Lebeck, Mundhra, and Thottethodi [1999], Valsalam and Skjellum [2002], and Frens and Wise [1997] achieve this by recursive nesting, which has the added advantage that there is no need to choose a block size. In [Waśniewski et al. 1998; Andersen et al. 2001; Andersen et al. 2002], the recursive blocking is applied to triangular matrices in full and packed storage format.

In designing the Level-3 BLAS, Dongarra, Du Croz, Duff, and Hammarling [1990] chose not to address packed storage schemes for symmetric, Hermitian or triangular matrices because 'such storage schemes do not seem to lend themselves to partitioning into blocks ... Also packed storage is required much less with large memory machines available today'. In this paper, our aim is to demonstrate that packing is possible without any loss of performance. While memories continue to get larger, problems that people solve get larger too and there will always be an advantage in saving storage.

We achieve this by using a blocked hybrid format in which each block is held contiguously in memory. It avoids the data copies that are inevitable when Level-3 BLAS are applied to matrices held conventionally in rectangular arrays. Note, too, that many data copies may be needed for the same submatrix in the course of a Cholesky factorization [Gustavson 1997].

The rest of the paper is organized as follows. Our proposed blocked hybrid format for a lower-triangular matrix is explained in Section 2. How Cholesky factorization and solution of equations can be implemented using this format is described in Sections 3 and 4, respectively. In Section 5, we consider the lower-triangular case 


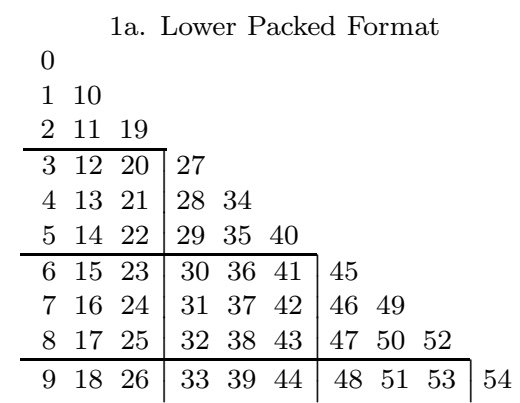

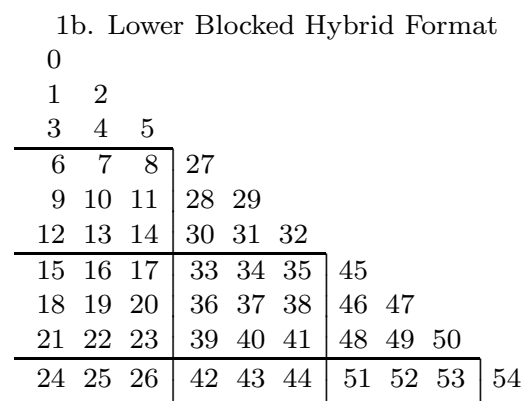

Fig. 1. Lower Packed and Blocked Hybrid Formats.

similarly. A kernel code for triangular factorization of the blocks on the diagonal of our blocked form is needed and we explain this in Section 6 . The results of performance testing, with comparisions against conventional full format (LAPACK) and the recursive format of Andersen, Gustavson, and Waśniewski, are given in Section 7. We also show the (much inferior) performance of the LAPACK code for packed format that relies on Level-2 BLAS. Our conclusions are drawn in Section 8.

\section{LOWER PACKED FORMATS}

The form of packed storage used by LINPACK [Dongarra et al. 1979] is that the upper-triangular part is held by columns. The Level-2 BLAS [Dongarra et al. 1988] and LAPACK [Anderson et al. 1999] permit the lower-triangular or uppertriangular part to be held by columns. We refer to these as the lower and upper packed formats, respectively. We show an example of the lower packed format in Figure 1a, with blocks of size 3 superimposed. In this and all the other figures that illustrate formats, we show where each matrix element is stored within the array that holds it.

It is apparent that the blocks are not suitable for passing to the BLAS since the stride between elements of a row is not uniform. We therefore propose to rearrange each trapezoidal block column so that it is stored by blocks with each block in row-major order, as illustrated in Figure 1b. Unless the order is an integer multiple of the block size, the final block will be shorter than the rest. Later in this section, we will explore alternative formats. We assume that the block size is chosen so that a block fits comfortably in level-1 cache. We defer discussion of the upper packed format to Section 5 .

If the matrix order is $n$ and the block size is $n b$, this rearrangement may be performed efficiently in place with the aid of a buffer of size $n \times n b$. For each block column, we copy the data to the buffer, reordering it so that it is held by rows in packed format, then copy the data back, overwriting the original block column. To reduce cache misses, the blocks are copied one by one, each copy being completed before the next is commenced. Within each block, we access the columns one by one and copy each to its new position. Little cache memory will be needed for the original columns and the whole block in the buffer should remain in the cache until its last column has been formed. On some machines, there is an efficiency gain 


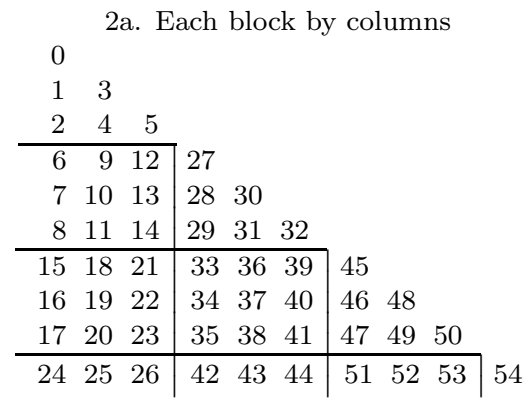

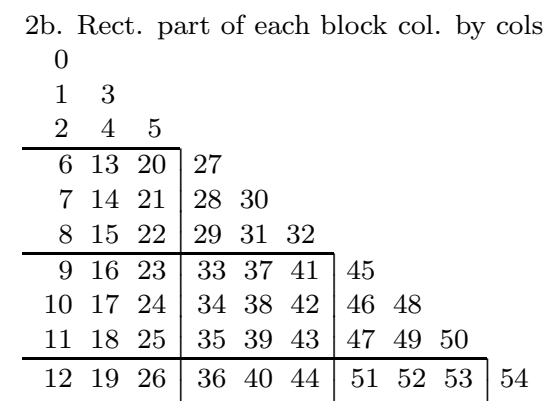

Fig. 2. Alternative Lower Blocked Formats.

from the use of the Level-1 BLAS _COPY [Lawson et al. 1979], which copies vectors with uniform strides and uses loop unrolling. The subroutine _COPY may be used directly for copying a column of a rectangular block to the buffer (with stride $n b$ in the buffer) and for copying the whole rearranged block column back from the buffer. We may use _COPY for the triangular block, too, by treating it separately; we expand the triangle to full form in the buffer and copy it back row by row; a simple copy may still be used for the rest of the block column.

It would have been easier to perform this rearrangement had we held each block by columns, as illustrated in Figure 2a, or the whole rectangular part of each block column by columns, as illustrated in Figure 2b and used by Duff and Reid [1996]. We explain in the next section why we expect that holding the blocks by columns will be less efficient.

We use the same block form for holding the Cholesky factor, which of course means that new codes are needed for forward and back substitution. We could have rearranged the matrix back to the packed format used by LINPACK and LAPACK, but the blocking is also helpful during forward and back substitution, particularly for multiple right-hand sides where the blocking allows the use of Level-3 BLAS.

\section{BLOCK CHOLESKY FACTORIZATION}

The Cholesky factorization of a symmetric and positive-definite matrix $A$ usually takes the form

$$
A=L L^{T}
$$

where $L$ is a lower-triangular matrix. If the same partitioning is applied to the rows and columns of $A$ and the rows and columns of $L$, the resulting blocks $A_{i j}$ and $L_{i j}$, with $i$ and $j$ in the range 1 to $l$, satisfy the relation

$$
A_{i j}=\sum_{k=1}^{j}\left(L_{i k} L_{j k}^{T}\right), \quad i \geq j
$$

which allows the diagonal blocks $L_{j j}$ of $L$ to be found from the equation

$$
L_{j j} L_{j j}^{T}=A_{j j}-\sum_{k=1}^{j-1}\left(L_{j k} L_{j k}^{T}\right)
$$




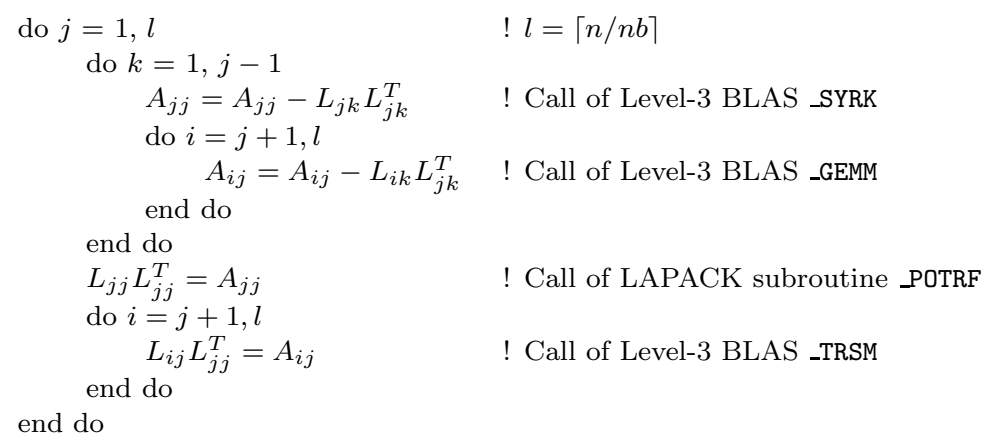

Fig. 3. $L L^{T}$ Implementation for Lower Blocked Hybrid Format. The BLAS calls take the forms

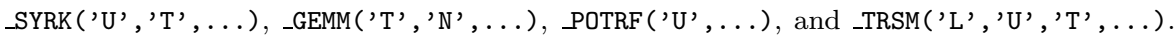

and the off-diagonal blocks $L_{i j}(i>j)$ to be found from the equation

$$
L_{i j} L_{j j}^{T}=A_{i j}-\sum_{k=1}^{j-1}\left(L_{i k} L_{j k}^{T}\right) .
$$

We will assume that the first $l-1$ blocks are of size $n b$ and that the final block is of size at most $n b$, so that $l=\lceil n / n b\rceil .^{1}$

We have some choice over the order in which these operations are performed and how they are grouped. For the lower blocked hybrid form, it is natural to calculate the blocks column by column as shown in Figure 3. Each of the computation lines in the figure can be implemented by a single call of a Level-3 BLAS or LAPACK subroutine and we show which as a comment. However, it may be better to make a direct call to an equivalent 'kernel' routine that is fast because it has been specially written for matrices that are held in contiguous memory and are of a form and size that permits efficient use of the level- 1 cache. We propose a recursive full storage format Cholesky subroutine in Section 6 for situations where a fast kernel is not available from the vendor or elsewhere.

For large problems, the most significant computation is that of _GEMM, which is performed on matrices held in contigous memory and is actually called for the transpose of the equation in Figure 3, that is,

$$
A_{i j}^{T}=A_{i j}^{T}-L_{j k} L_{i k}^{T} .
$$

Note that $L_{j k}$ is held by rows and $A_{i j}^{T}$ and $L_{i k}^{T}$ are held by columns. The operation can therefore be applied efficiently with streaming, as explained in Section 1 , without any data rearrangement. This is why we have not chosen either of the alternative formats of Figure 2. Similarly, the format of Figure $1 \mathrm{~b}$ is favourable for the call of _SYRK; in fact, $L_{j k}$ should remain in cache for the call of _SYRK and all the calls of _GEMM in the loop that follows. Similarly, the array that holds first $A_{j j}$ and then $L_{j j}$ will remain resident in the cache during the factorization of $A_{j j}$ by _POTRF and throughout the _TRSM call, during which the rows are streamed into the cache as $A_{i j}$ and out of the cache as $L_{i j}$.

\footnotetext{
${ }^{1}$ The notation $\lceil x\rceil$ refers to the least integer $i \geq x$, that is, the ceiling function.
} 


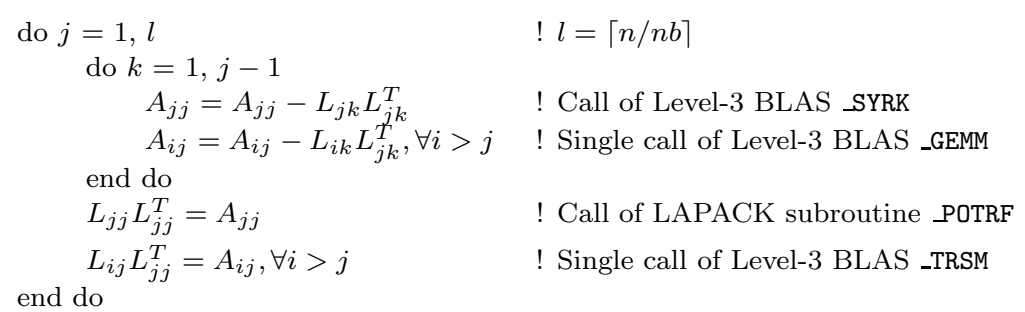

Fig. 4. $L L^{T}$ Implementation for Lower Blocked Hybrid Format with BLAS Called Once for Each Block Column. The BLAS calls take the forms _SYRK('U', 'T', ...), _GEMM('T', 'N', ...), POTRF ('U', $\ldots$ ), and _TRSM('L', 'U', 'T', ...).

The fact that our blocks are held contiguously is significant. Without this (see, for example, Figure 2b) either the cache is used less efficiently with unneeded data being brought in, or a preliminary rearrangement is needed.

For the operations with $A_{j j}$, use of the subroutines _SYRK and _POTRF requires that a temporary full-format copy of $A_{j j}$ be made at the beginning of the main loop. POTRF overwrites this by $L_{j j}$, which is used by _TRSM and then packed to overwrite $A_{j j}$. A buffer of size $n b \times n b$ is needed for the full-format copy.

A further merit of the lower blocked hybrid format (Figure 1b) is that all the operations

$$
A_{i j}=A_{i j}-L_{i k} L_{j k}^{T}, \forall i>j
$$

may be performed in a single call of _GEMM that involves multiplying a matrix of order $n-j \times n b$ by $n b$ by a matrix of order $n b$ by $n b$. This is possible since the whole trapezoidal block column is held by rows, which means that all the rectangular blocks of the block column can be passed as a single matrix to _GEMM. Similarly, the equation

$$
L_{i j} L_{j j}^{T}=A_{i j}, \forall i>j
$$

may be solved with a single call of _TRSM for a matrix of order $n-j \times n b$ by $n b$. We summarize the resulting code in Figure 4. The data format allows both _GEMM and _TRSM to perform their operations with streaming and without data copying, but we have no guarantee that library versions that are written for more general situations will do this.

The LAPACK subroutine _POTRF performs block Cholesky factorization of a symmetric positive-definite matrix held in full format (see LAPACK Users' Guide [Anderson et al. 1999, pages 29 and 295]). We show in Figure 5 how _POTRF is organized. The subroutine _POTF2 is an unblocked version of POTRF. Note that Figures 4 and 5 are very similar. The difference is that the inner do loop of Figure 4 has been replaced by single calls of _SYRK and _GEMM, possible with the full format since any off-diagonal submatrix can be passed directly to a Level-3 BLAS as a rectangular matrix. For a large problem, most of the work is done by _GEMM and passing it a bigger problem gives it more scope for optimization. However, there is the disadvantage that the matrix that is sent to it is not in contiguous memory. It is our belief that most implementations of Level-3 BLAS begin with a data copy for each block of each operand to put it in contiguous memory and for each block of the 


$$
\begin{aligned}
\text { do } j=1, l & ! l=\lceil n / n b\rceil \\
A_{j j}=A_{j j}-\sum_{k=1}^{j-1}\left(L_{j k} L_{j k}^{T}\right) & \text { ! Single call of Level-3 BLAS _SYRK } \\
L_{j j} L_{j j}^{T}=A_{j j} & \text { ! Call of LAPACK subroutine _POTF2 } \\
A_{i j}=A_{i j}-\sum_{k=1}^{j-1}\left(L_{i k} L_{j k}^{T}\right), \forall i>j & \text { ! Single call of Level-3 BLAS _GEMM } \\
L_{i j} L_{j j}^{T}=A_{i j}, \forall i>j & \text { ! Single call of Level-3 BLAS _TRSM }
\end{aligned}
$$

Fig. 5. LAPACK Cholesky Implementation for Lower Full Format (POTRF). The BLAS

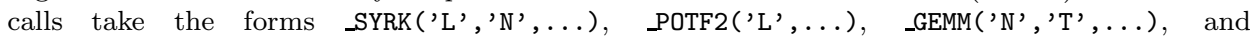
_TRSM('R', 'L' , 'T', ...).

result to return it to its original format. For this reason, we expect that the code for the blocked hybrid format will usually be faster (as well as needing about half the memory).

\section{SOLVING EQUATIONS USING A BLOCK CHOLESKY FACTORIZATION}

Given the Cholesky factorization (2), we may solve the equations

$$
A X=B
$$

by forward substitution

$$
L Y=B
$$

followed by back-substitution

$$
L^{T} X=Y
$$

If the rows of $B, Y$, and $X$ are partitioned in the same way as $A$ and $L$, equations (7) and (8) take the form

$$
L_{i i} Y_{i}=B_{i}-\sum_{j=1}^{i-1}\left(L_{i j} Y_{j}\right), i=1,2, \ldots, l
$$

and

$$
L_{j j}^{T} X_{j}=Y_{j}-\sum_{i=j+1}^{l}\left(L_{i j}^{T} X_{i}\right), j=l, l-1, \ldots, 1,
$$

where $l=\lceil n / n b\rceil$.

We begin by discussing the important special case where $B$ has only one column. This, of course, means that $B_{i}, Y_{i}$, and $X_{i}$ are all blocks that have a single column. If $L$ is held in lower blocked hybrid format, the forward substitution may be performed by subtracting $L_{i j} Y_{j}$ from $B_{i}$ as soon as $Y_{j}$ is available using a single call of _GEMV, as shown in the first part of Figure 6 . The back-substitution can be performed with a single call of _GEMV for each summation, as shown in the second part of Figure 6. The whole of $L$ must be accessed once during forward substitution and once during back-substitution and in both cases the whole of the rectangular part of each block column is accessed in a single call of _GEMV.

Similar code could be applied in the case where $B$ has $m>1$ columns, with _TRSM replacing _TPSV (this will require making a copy of $L_{j j}$ in full storage) and _GEMM 


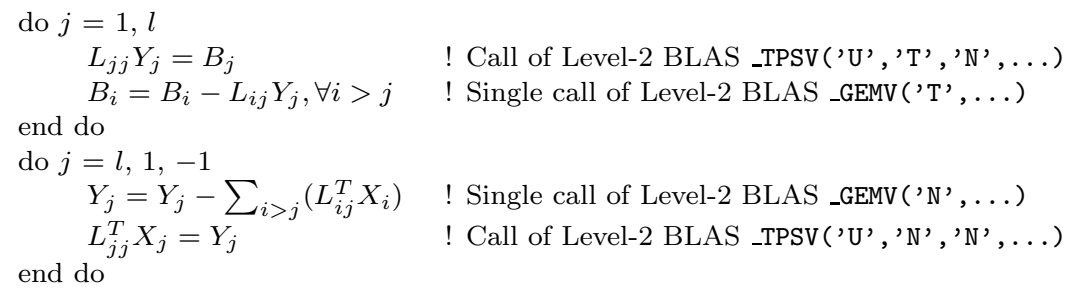

Fig. 6. Forward substitution and back-substitution for a single right-hand side using Lower Blocked Hybrid Factorization $L L^{T}$.

\begin{tabular}{rrrr}
0 & 3 & 6 & 9 \\
1 & 4 & 7 & 10 \\
2 & 5 & 8 & 11 \\
\hline 12 & 15 & 18 & 21 \\
13 & 16 & 19 & 22 \\
14 & 17 & 20 & 23 \\
\hline 24 & 27 & 30 & 33 \\
25 & 28 & 31 & 34 \\
26 & 29 & 32 & 35 \\
\hline 36 & 37 & 38 & 39
\end{tabular}

Fig. 7. The blocked format for $B$.

replacing _GEMV. However, the blocks $B_{i}, Y_{i}$, and $X_{i}$ would not occupy contiguous memory, so there is scope for improving the performance.

If the number of columns $m$ is modest, we therefore make a copy of $B$ as a block matrix, with each block $B_{i}$ held contiguously by columns and the blocks held contiguously, as illustrated in Figure 7.

Code for forward substitution and back-substitution is shown in Figure 8. Each of the operations $L_{i j} Y_{j}$ and $L_{i j}^{T} X_{i}$ is performed by a separate call of _GEMM, but each submatrix involved is held contiguously and streaming is available. Finally, $X$ is copied back to overwrite the original $B$.

The overhead of copying all the diagonal blocks $L_{j j}$ to full storage may be halved

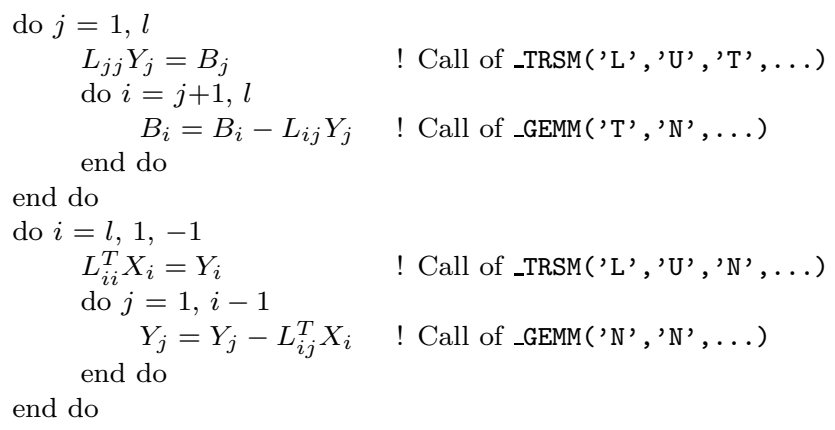

Fig. 8. Forward substitution and back-substitution for many right-hand sides using Lower Blocked Hybrid Factorization $L L^{T}$. 
if we have a buffer that is big enough to hold them all, so that they are already available for the back-substitution. The size needs to be $n \times n b$, which is the same as we used in Section 2. Of course, this means that we need two buffers, one of size $n \times n b$ for the diagonal blocks and one of size $n \times m$ for the right-hand sides.

For very small numbers of columns, say 2 or 3 , the copying overhead probably means that it is better to handle each column separately, as in Figure 6.

For very large numbers of columns, we simply apply the same process of forward substitution and back-substitution (Figure 8) to successive blocks of columns. In our code, we allow for a different block size $m b$. Buffers of total size $n \times(n b+m b)$ are needed. Note that with two buffers, the overhead of copying the diagonal blocks is incurred only once for all the forward substitutions and back-substitutions on all the block columns.

For very big problems, the block size $m b$ is probably best chosen to be similar to $n b$ for two reasons. The first is simply that making it much bigger would substantially increase the total buffer size. The second is that for a very big problem the factorized matrix has to be moved from memory or level-3 cache for each block of $m b$ columns, a total data movement of about $n^{2} \frac{m}{m b}$ reals. Meanwhile, if $n \times m b$ is too big for level-2 cache, the data movement for the right-hand side averages at about $\frac{n}{2} \times m b$ reals for each block step of the forward or back substitution to give a total of about $(n \times m b) \times \frac{n}{n b} \times \frac{m}{m b}=n^{2} \frac{m}{n b}$ reals. It follows that the data movement is balanced with $m b=n b$ and will be increased if either is $m b$ or $n b$ is increased at the expense of the other.

If the problem is big but not such that the level- 2 cache cannot hold $n \times n b$ reals, there are likely to be performance advantages in chosing the block size $m b$ to be larger than $n b$ (or even equal to $m$ ). This is because each of the blocks of the factorized matrix is accessed once for each block column of $B$, so increasing $m b$ reduces level-2 cache movement provided there is still room in level-2 cache for $n \times m b$ reals.

9a. Upper Packed Format

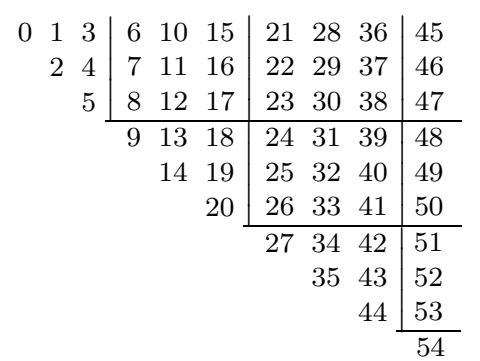

9b. Upper Blocked Hybrid Format

\begin{tabular}{rrr|rrr|rrr|r}
0 & 1 & 3 & 6 & 9 & 12 & 21 & 24 & 27 & 45 \\
& 2 & 4 & 7 & 10 & 13 & 22 & 25 & 28 & 46 \\
& & 5 & 8 & 11 & 14 & 23 & 26 & 29 & 47 \\
\hline & & 15 & 16 & 18 & 30 & 33 & 36 & 48 \\
& & & 17 & 19 & 31 & 34 & 37 & 49 \\
& & & & 20 & 32 & 35 & 38 & 50 \\
\hline & & & & & 39 & 40 & 42 & 51 \\
& & & & & & 41 & 43 & 52 \\
& & & & & & & & 44 & 53 \\
& & & & & & & & &
\end{tabular}

Fig. 9. Upper Packed and Blocked Hybrid Formats.

\section{UPPER PACKED FORMATS}

We will now consider the Cholesky factorization of a matrix in upper packed format (see Figure 9a). Our chosen blocked hybrid format is illustrated in Figure 9b. It is ordered by block columns, which allows us to get the same desirable properties 


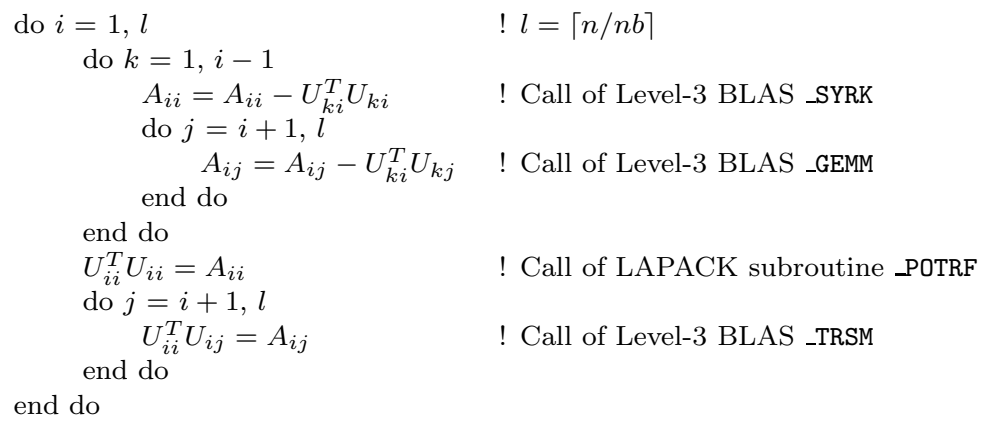

Fig. 10. $U^{T} U$ Cholesky Implementation for Upper Blocked Hybrid Format. The BLAS calls take the forms _SYRK('U', 'T',..), _GEMM('T', 'N', ...), POTRF('U',..), and _TRSM('L', 'U', ' T', ,...).

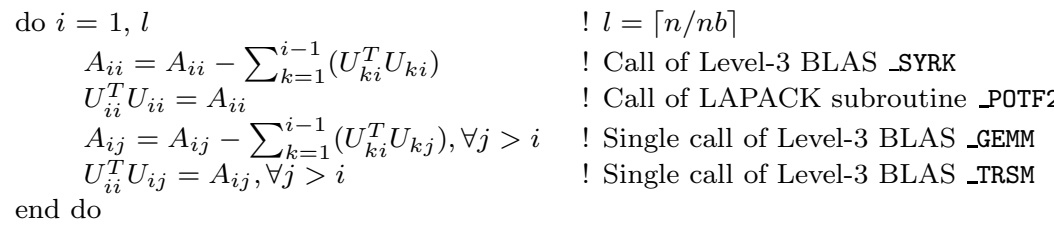

Fig. 11. LAPACK Cholesky Implementation for Upper Full Format. The BLAS calls take the forms _SYRK ('U' , 'T', ...), POTF2 ('U', ...), _GEMM(' 'T', 'N',$\ldots$ ), and _TRSM(' $L$ ' , 'U' , 'T', ...).

for the rearrangement that we had for the lower packed format (Section 2). The individual blocks are ordered by columns to permit efficient calls of Level-3 BLAS. A consequence is that the rearrangement code runs a little faster than for the lower packed hybrid format since the entries of each column of each block are contiguous before and after rearrangement.

LAPACK returns the matrix $U$ of the $U^{T} U$ factorization, so we first consider how this may be done with a blocked hybrid format. We then consider the alternative of using a backwards pivot sequence, that is, performing a $U U^{T}$ factorization. From the error analysis point of view, this is equally satisfactory; both are unconditionally stable for a symmetric positive-definite matrix. The $U U^{T}$ factorization has performance advantages, but is unsuitable if compatibility with LAPACK is needed.

The algorithm we have chosen may be obtained by transposing every matrix in Figure 3 and interchanging $i$ with $j$ to obtain Figure 10. The outer loop is over block rows and within it there is a block outer product. Note also the similarity with the way the LAPACK subroutine _POTRF performs a block $U^{T} U$ factorization of a matrix held in upper full format, see Figure 11.

If we use the upper blocked hybrid format of Figure 9b, we now get all the same desirable properties for level-1 cache usage, but we cannot combine the _GEMM calls in the inner loop into one call since the blocks are not held contiguously. Note, however, that the same block $U_{k i}^{T}$ is used for each call in the inner loop, so this will remain resident in level-1 cache for all the calls in the inner loop. Similarly, 
the _TRSM calls cannot be merged, but the block $U_{i i}^{T}$ will remain resident in level-1 cache.

We anticipate that the effect of the blocks not being contiguous in memory will be quite minor and this is borne out by the results in Section 7, where we see that the code for the upper packed hybrid format is only sightly slower than that for the lower packed hybrid format. There are more procedure calls, but each does a significant amount of work, so the call itself is a small overhead. There will also be some unnecessary data movement to and from the cache at the edges of the blocks, but this will be small compared with that needed for the blocks themselves. Finally, if the level-2 cache is not big enough for the whole matrix but is big enough (with a reasonable margin) for a block row, the pivot block row will be resident in level-2 cache for all the operations associated with the block pivot even though the blocks are not contiguous.

To get contiguous blocks, we considered rearranging the blocks so that they are held by block rows, see Figure 12a. Actually, this rearranged form is also the lower blocked hybrid format representation of the matrix (see Figure 1b), since any ordering of the upper triangular entries $a_{i j}, i \leq j$, of a symmetric matrix $A$ is also an ordering of its lower triangular entries $a_{j i}, i \leq j$. Hence, if we did this rearrangement, we could apply a code implementing the algorithm of Figure 3 and thereby get all the desirable properties of that algorithm. However, we rejected this approach because of the difficulty of constructing an efficient in-place code for rearrangement. The best rearrangement algorithm that we found requires an additional integer array of length the total number of blocks and we estimate that it would run about twice as slowly as our present rearrangement code.

12a. The Blocks Ordered by Rows

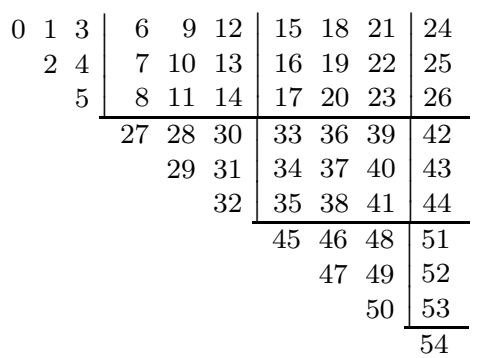

12b. Each Block Ordered by Rows

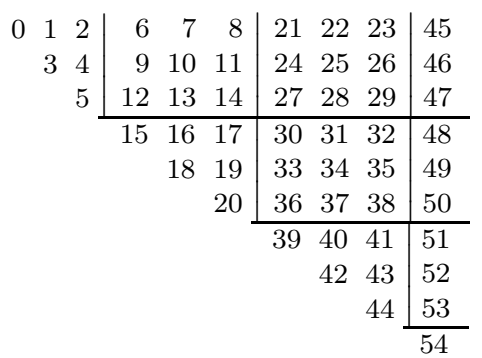

Fig. 12. Alternative Upper Blocked Hybrid Formats.

For solving sets of equations, similar considerations apply for the upper blocked hybrid format to those discussed in Section 4. The code for many right-hand sides is illustrated in Figure 13. The code for a single right-hand side is very similar, again with _GEMM replaced by _GEMV and _TRSM replaced by _TPSV. Note that we are not able to combine calls of _GEMV with this data format.

If it is acceptable to reverse the pivot order, that is perform a $U U^{T}$ factorization

$$
A=U U^{T}
$$

where $U$ is upper triangular, we can get the desirable properties without an additional rearrangement. Now we have the relation 


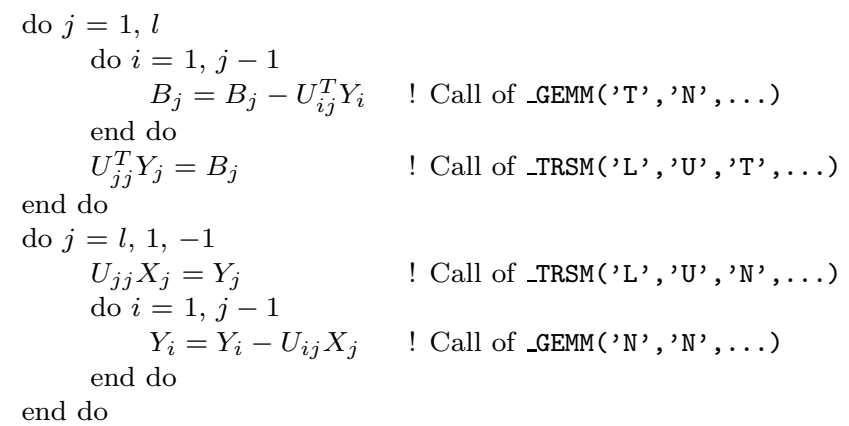

Fig. 13. Forward substitution and back-substitution for many right-hand sides using Upper Blocked Hybrid Factorization $U^{T} U$.

$$
A_{i j}=\sum_{k=j}^{l}\left(U_{i k} U_{j k}^{T}\right), \quad i \leq j
$$

which allows the diagonal blocks $U_{j j}$ to be found from the equation

$$
U_{j j} U_{j j}^{T}=A_{j j}-\sum_{k=j+1}^{l}\left(U_{j k} U_{j k}^{T}\right)
$$

and the off-diagonal blocks $U_{i j}(i<j)$ to be found from the equation

$$
U_{i j} U_{j j}^{T}=A_{i j}-\sum_{k=j+1}^{l}\left(U_{i k} U_{j k}^{T}\right) .
$$

We now need to loop through the block columns in reverse order, as illustrated in Figure 14. Again, each of the computation lines in the figure can be implemented by a single call of a Level-3 BLAS or LAPACK subroutine or an equivalent kernel routine and we show which as a comment. If each block is held by rows, see Figure $12 \mathrm{~b}$, we get all the desirable properties of the Figure 3 algorithm for level-1 cache usage and we can merge the BLAS calls in the inner loops into single calls. There is the disadvange that we will need to permute the diagonal block $A_{j j}$ before passing it to _POTRF and permute the factor when packing it back.

For the use of a $U U^{T}$ factorization to solve a set of equations, similar considerations apply for the upper blocked hybrid format to those discussed in Section 4. Here, we need to perform a back-substitution followed by a forward substitution, see Figure 15.

The disadvantage of having to permute the diagonal block may be avoided by reversing the order within each block and of the blocks within each block column. We do not reverse the order of the block columns themselves since this does not impact the efficiency of the algorithm and allows the rearrangement to blocked hybrid format to be performed independently for each block column. The format is illustrated in Figure 16a. 


$$
\begin{array}{cl}
\text { do } j=l, 1,-1 & ! l=\lceil n / n b\rceil \\
\text { do } k=j+1, l & \text { ! Call of Level-3 BLAS _SYRK } \\
A_{j j}=A_{j j}-U_{j k} U_{j k}^{T} & \\
\text { do } i=1, j-1 & \\
A_{i j}=A_{i j}-U_{i k} U_{j k}^{T} & \text { ! Call of Level-3 BLAS _GEMM } \\
\text { end do } & \\
\text { end do } & \text { ! Call of LAPACK subroutine _POTRF } \\
\begin{array}{l}
U_{j j} U_{j j}^{T}=A_{j j} \\
\text { do } i=1, j-1
\end{array} & \text { ! Call of Level-3 BLAS _TRSM } \\
U_{i j} U_{j j}^{T}=A_{i j} &
\end{array}
$$

Fig. 14. $U U^{T}$ Implementation for Upper Blocked Hybrid Format. With the format of Figure $12 \mathrm{~b}$, the BLAS calls take the forms _SYRK (' $\mathrm{L}$ ' , ' $T$ ',$\ldots$ ), _GEMM(' $T$ ' , ' $N$ ',$\ldots$ ), POTRF ('L', ...), and _TRSM('L', 'L', 'T', ...).

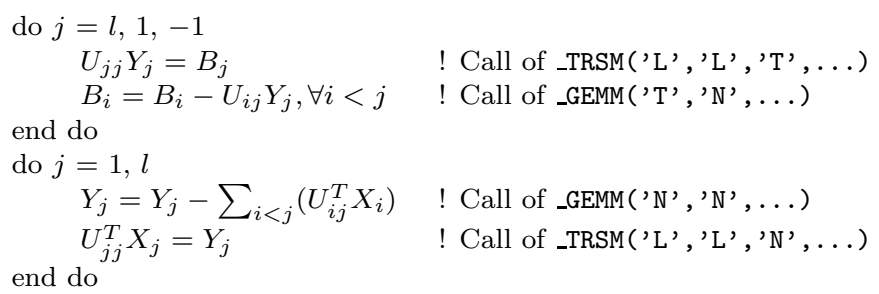

\begin{tabular}{|c|c|c|c|c|c|c|c|}
\hline $\begin{array}{ll}98 \\
\end{array}$ & 87 & 27 & 26 & 25 & 54 & 53 & 52 \\
\hline & $\begin{array}{ll}54 \\
\end{array}$ & 24 & 23 & 22 & 51 & 50 & 49 \\
\hline & 32 & 21 & 20 & 19 & 48 & 47 & 46 \\
\hline & 1 & 18 & 17 & 16 & 45 & 44 & 43 \\
\hline & & 15 & 14 & 13 & $\overline{42}$ & 41 & 40 \\
\hline & & & 12 & 11 & 39 & 38 & 37 \\
\hline & & & & 10 & 36 & 35 & 34 \\
\hline & & & & & 33 & 32 & 31 \\
\hline & & & & & & 30 & 29 \\
\hline & & & & & & & 28 \\
\hline
\end{tabular}

Fig. 15. Back-substitution and forward substitution for many right-hand sides using Upper Blocked Hybrid Factorization $U U^{T}$ with the format of Figure $12 \mathrm{~b}$.

16a. The Ordering 16b. When Rotated by 180 degrees

28

2930

$\begin{array}{lll}31 & 32 & 33\end{array}$

\begin{tabular}{lll|l}
\hline 34 & 35 & 36 & 10
\end{tabular}

$\begin{array}{lllll}37 & 38 & 39 & 11 & 12\end{array}$

\begin{tabular}{lll|lll}
40 & 41 & 42 & 13 & 14 & 15
\end{tabular}

\begin{tabular}{lll|lll|l}
\hline 43 & 44 & 45 & 16 & 17 & 18 & 1
\end{tabular}

\begin{tabular}{llllll|lll}
46 & 47 & 48 & 19 & 20 & 21 & 2 & 3
\end{tabular}

\begin{tabular}{lll|lll|lll|l}
49 & 50 & 51 & 22 & 23 & 24 & 4 & 5 & 6 \\
\hline 52 & 53 & 54 & 25 & 26 & 27 & 7 & 8 & 9 & 0
\end{tabular}

Fig. 16. Another Upper Blocked Hybrid Format, obtained by reversing the order within each block and of the blocks within each block column. 
Figure 16b depicts Figure 16a rotated by 180 degrees and shows how it represents the matrix $P A P^{T}$ for $P$ the permutation that reverses the order. Figure $16 \mathrm{~b}$ is closely related to Figure $1 \mathrm{~b}$. The only difference is that the order of the trapezoidal block columns is reversed. If the offsets $-28,17,44,54$ are applied to all locations in block columns 1, 2, 3, and 4, respectively, of Figure 16b, we obtain Figure 1b. This means that we can apply the algorithm depicted in Figure 3 to $P A P^{T}$ by adding the appropriate offset to all the addresses in each block column. And for forward and back substitution, the code in Figure 8 will work by adding the appropriate offset to all addresses in each block column.

\section{LEVEL-3 CHOLESKY KERNEL SUBROUTINES}

For each of our block factorizations (see Figures 3, 4, 10, and 14), we have employed the LAPACK subroutine POTRF to factorize the diagonal blocks, held in upper full format. This is itself a block algorithm and is summarized in Figure 11. We expect its block size to be about the same as ours. This means that a significant proportion of its computation, or perhaps all of it, is performed by POTF2. This is unsatisfactory since it uses Level-2 BLAS. The purpose of this section is to consider alternatives that use Level-3 BLAS.

In this section, we still use the notation $A$ for the matrix, $n$ for its order, and $L L^{T}$ and $U^{T} U$ for its Cholesky factorizations, but these refer to a diagonal block of the overall computation. This should be no confusion since the discussion is confined to factorizing a block. If should be borne in mind that $n$ will be modest, small enough for the computation to reside in level-1 cache.

The subroutine _POTRF uses full storage mode, that is, it requires $n^{2}$ memory locations even though it only access $n(n+1) / 2$ matrix elements. The rest of the locations are not used. We have chosen to follow this for our alternatives because all our algorithms rely on _SYRK and _TRSM and because it allows Level-3 BLAS to be used on submatrices without any further data movement.

Recently, four papers focusing on recursive Cholesky algorithms were published, [Gustavson 1997], [Waśniewski et al. 1998], [Gustavson and Jonsson 2000], and [Andersen et al. 2001]. These papers demonstrate that full storage data format recursive Cholesky algorithms can be developed using Level-3 BLAS for almost all the computation. The appendices of the first and fourth papers contain Fortran 77 and Fortran 90 implementations of the algorithms.

For the upper packed format, these recursive algorithms rely on partitioning the matrices $A$ and $U$ into submatrices:

$$
A=\left(\begin{array}{ll}
A_{11} & A_{12} \\
A_{12}^{T} & A_{22}
\end{array}\right) \text { and } U=\left(\begin{array}{cc}
U_{11} & U_{12} \\
& U_{22}
\end{array}\right),
$$

where $A_{11}$ and $U_{11}$ are $p \times p$ and $p=\lfloor n / 2\rfloor$. Of course, only the upper-triangular parts of $A_{11}$ and $A_{22}$ are stored. The matrices $U_{11}$ and $U_{22}$ are upper triangular and the matrices $A_{12}$ and $U_{12}$ are square or nearly square (of size $p \times n-p$ ).

We arrive at a recursive algorithm by simple algebraic manipulations on the partitioning indicated above, see Figure 17. Note that the only floating-point operations that are performed outside calls to _TRSM and _SYRK are the calculation of $n$ square roots.

In Figure 17, the recursion continues all the way down to the single diagonal 


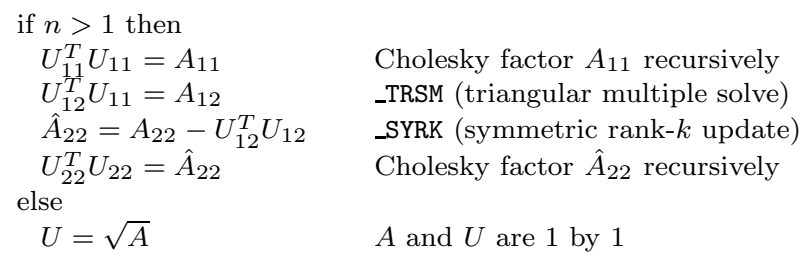

Fig. 17. Recursive Cholesky Kernel.

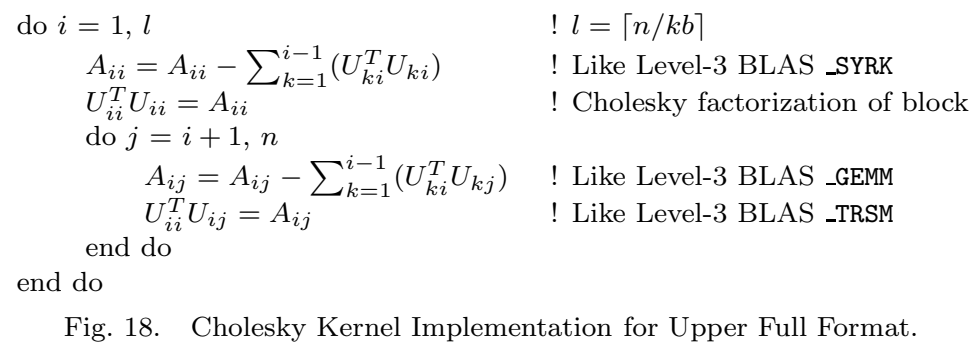

elements. During the factorization of very small matrices close to the end of the recursion, there are very few floating-point operations in comparison to the number of subroutine calls, administrative, and fixed-point calculations in the recursively called subroutine. This makes the factorization relatively inefficient. A way to alleviate this inefficiency is to inline the code for these small matrices; for instance, for matrices of size up to $4 \times 4$. The first line of Figure 17 must be replaced by

$$
\text { if } n>4 \text { then }
$$

and the last line by the Cholesky factorization of $A$, using special unrolled code.

By choosing the recursive division of the coefficient matrix differently, it is possible to make sure that the final factorization at the leaves of the recursion is a $4 \times 4$ matrix in all cases except the final leaf. This is done by choosing $p$ always to be a multiple of 4 . It means that a special inlined code can be used for the $4 \times 4$ case. Of course, when $n$ is not a multiple of 4 , there must be some code that handles a single block that is smaller than $4 \times 4$.

Another possibility is to use a block algorithm with a very small block size $k b$, designed to fit in registers. To avoid procedure call overheads for a very small computations, we replace all calls to BLAS by in-line code. This means that it is not advantageous to perform a whole block row of _GEMM updates at once and a whole block row of _TRSM updates at once (see last two lines of the loop in Figure 11) . This leads to the algorithm summarized in Figure 18.

We have found the block size $k p=2$ to be suitable. The key loop is the one that corresponds to _GEMM. For this, the code of Figure 19 is suitable. The block $A_{i, j}$ is held in the four variables, $\mathrm{t} 11, \mathrm{t} 12, \mathrm{t} 21$, and $\mathrm{t} 22$. We reference the underlying array directly, with $A_{i, j}$ held from a $(i i, j j)$. It may be seen that a total of 8 local variables are involved, which hopefully the compiler will arrange to be held in registers. The loop involves 4 memory accesses and 8 floating-point operations.

On some processors, faster execution is possible by having an inner _GEMM loop 


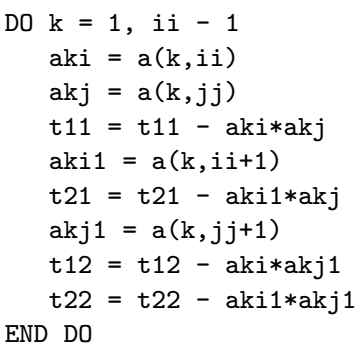

Fig. 19. Code corresponding to _GEMM.

Table 1. Computers Used. The IBM level-2 and level-3 caches are shared between two processors.

\begin{tabular}{lcccccc}
\hline Processor & MHz & Peak & \multicolumn{3}{c}{ Cache sizes } & TLB \\
& & Mflops & level-1 & level-2 & level-3 & entries \\
\hline IBM Power4 & 1700 & 6800 & $64 \mathrm{~K}$ & $1.5 \mathrm{M}^{*}$ & $32 \mathrm{M}^{*}$ & 1024 \\
SUN UltraSparc IIICu & 900 & 1800 & $64 \mathrm{~K}$ & $8 \mathrm{M}$ & None & 512 \\
SGI MIPS R12000 & 300 & 600 & $32 \mathrm{~K}$ & $8 \mathrm{M}$ & None & 64 \\
HP Alpha EV6 & 500 & 1000 & $64 \mathrm{~K}$ & $4 \mathrm{M}$ & None & 128 \\
HP Itanium 2 & 1000 & 4000 & $32 \mathrm{~K}$ & $256 \mathrm{~K}$ & $1.5 \mathrm{M}$ & 128 \\
INTEL Pentium III & 500 & 500 & $16 \mathrm{~K}$ & $512 \mathrm{~K}$ & None & 32 \\
\hline
\end{tabular}

that updates $A_{i, j}$ and $A_{i, j+1}$. The variables aki and aki1 need only be loaded once, so we now have 6 memory accesses and 16 floating-point operations and need 14 local variables, hopefully in registers. We found that this algorithm gave very good performance (see next section).

We will make our implementation of this kernel available in a companion paper, but alternatives should be considered. A version of a recursive Cholesky algorithm was programmed and added to the ATLAS library by [Whaley et al. 2000]. The LAPACK library can be updated to use this new recursive subroutine by using a simple script from the ATLAS subdirectory (ATLAS/doc/LAPACK.txt; the ATLAS library can be found on http://www.netlib.org/atlas/). Further, every computer hardware vendor is interested in having good and well-tuned software libraries.

We recommend that all the alternatives of the previous paragragh be compared. Our kernel routine is available if the user is not able to perform such a comparison procedure or has no time to do it. Finally, note that LAPACK, ATLAS and the development of computer vendor software are ongoing activities. The implementation that is the slowest today might be the fastest tomorrow.

\section{PERFORMANCE}

For our performance testing, we have used the computers listed in Table 1 with the compilers listed in Table 2 and the libraries listed in Table 3.

In normal running, the shared level-2 cache on the IBM resulted in speeds that varied by up to about $30 \%$ according to the activity of the sharing processor. This made it impossible to conduct detailed comparisons between the algorithms. We therefore ran on a quiet machine, which means that the speeds we report are 
Table 2. Compilers Used.

\begin{tabular}{lll}
\hline Processor & Compiler & Option \\
\hline IBM Power4 & F95, version 8.1 & -05 \\
SUN UltraSparc IIICu & F95, Forte 7.1 & -fast -xarch=v8plusb \\
& & -xchip=ultra3 -free \\
SGI MIPS R12000 & F95, version, 7.3 & -03 -64 -freeform \\
HP Alpha EV6 & F95, version 5.3-915 & -free -0 \\
HP Itanium 2 & F95, version v2.7 & - -03 \\
Intel Pentium III & NAG F95, 4.1(340) & -free -04 \\
\hline
\end{tabular}

Table 3. Computer Libraries Used.

\begin{tabular}{ll}
\hline Processor & Library \\
\hline IBM Power4 & $\begin{array}{l}\text { ESSL, version 3.3; LAPACK routines } \\
\text { for_PPTRF and _PPTRS }\end{array}$ \\
SUN UltraSparc IIICu & Sun Performance Library 4.0 \\
SGI MIPS R12000 & SGI Scientific Library, 7.3.1.2 \\
HP Alpha EV6 & CXML Extended Math Library V3.6 \\
HP Itanium 2 & HP MLIB BLAS Library \\
Intel Pentium III & ATLAS, version 3.0 \\
\hline
\end{tabular}

optimistic for normal runs.

\subsection{Kernels}

We begin by considering the alternatives for the Cholesky kernel subroutine (Section 6). We consider orders 40, 72, and 100 since these will typically allow the computation to fit comfortably in level-1 cache. For each computer, we have compared

- the optimized LAPACK implementation provided by the vendor, if available, or otherwise the ATLAS optimized code;

- the published LAPACK source code compiled with the highest level of optimization;

- the recursive code of Section 6 ;

- the mini-blocked code of the end of Section 6 with all blocks of size $2 \times 2$; and

- the mini-blocked code of the end of Section 6 with blocks of sizes $2 \times 2$ and $2 \times 4$.

It may be seen from the results in Table 4 that the mini-blocked code with blocks of sizes $2 \times 2$ and $2 \times 4$ is remarkably successful. In all six cases, it significantly outperforms the compiled LAPACK code and the recursive algorithm. It outperforms the vendor's optimized codes except on the IBM platform at order 100. If compared with the mini-blocked code with all blocks of size $2 \times 2$, the performance is significantly better on the SUN (about 20\% times better except at order 72), slightly better on the Alpha (about 15\% times better), and much the same on the other four.

For our remaining performance testing, we use the vendor's optimized kernel on the IBM and the mini-blocked code with blocks of sizes $2 \times 2$ and $2 \times 4$ on the others. 
Table 4. Performance in Mflops of the Kernel Cholesky Algorithm. Comparison between different computers and different versions of subroutines.

\begin{tabular}{|c|c|c|c|c|c|}
\hline \multirow[t]{2}{*}{ Order } & \multicolumn{2}{|c|}{ LAPACK } & \multirow{2}{*}{$\begin{array}{r}\text { Recur- } \\
\text { sive }\end{array}$} & \multicolumn{2}{|c|}{ Mini-block } \\
\hline & Vendor & Comp. & & $2 \times 2$ & $\begin{array}{r}2 \times 2 \\
\& 2 \times 4\end{array}$ \\
\hline \multicolumn{6}{|c|}{ IBM Power4, 1700 MHz, ESSL Library: } \\
\hline 40 & 1658 & 1503 & 707 & 1999 & 1999 \\
\hline 72 & 2653 & 2303 & 1447 & 2751 & 2753 \\
\hline 100 & 3037 & 2481 & 1930 & 2957 & 2945 \\
\hline \multicolumn{6}{|c|}{ SUN Ultra III, $900 \mathrm{MHz}$, Sunperf BLAS Library: } \\
\hline 40 & 392 & 427 & 251 & 803 & 941 \\
\hline 72 & 598 & 664 & 417 & 1191 & 1012 \\
\hline 100 & 619 & 830 & 589 & 1143 & 1506 \\
\hline \multicolumn{6}{|c|}{ SGI Origin 2000, R12000, $300 \mathrm{MHz}$, Math Library: } \\
\hline 40 & 117 & 121 & 94 & 369 & 372 \\
\hline 72 & 197 & 212 & 166 & 455 & 466 \\
\hline 100 & 238 & 289 & 217 & 485 & 496 \\
\hline \multicolumn{6}{|c|}{ Alpha EV6, $500 \mathrm{MHz}$, DXML Library: } \\
\hline 40 & 311 & 313 & 165 & 457 & 533 \\
\hline 72 & 340 & 343 & 250 & 523 & 625 \\
\hline 100 & 393 & 400 & 323 & 551 & 647 \\
\hline \multicolumn{6}{|c|}{ HP Itanium 2, $1000 \mathrm{MHz}$, HP MLIB BLAS Library: } \\
\hline 40 & 449 & 448 & 153 & 1125 & 1133 \\
\hline 72 & 597 & 595 & 266 & 1711 & 1722 \\
\hline 100 & 567 & 559 & 364 & 2103 & 2103 \\
\hline \multicolumn{6}{|c|}{ Intel Pentium III, $500 \mathrm{MHz}$, ATLAS BLAS Library: } \\
\hline 40 & 83 & 97 & 86 & 177 & 169 \\
\hline 72 & 138 & 148 & 132 & 193 & 184 \\
\hline 100 & 157 & 155 & 147 & 182 & 179 \\
\hline
\end{tabular}

\subsection{Factorization}

The LAPACK subroutine _PPTRF performs Cholesky factorization of a symmetric positive-definite matrix held in unblocked packed format and the LAPACK subroutine PPTRS solves corresponding sets of equations using the factorization (see LAPACK Users' Guide [Anderson et al. 1999, pages 29 and 301]). Equations (2) to (5) remain applicable if we take all the blocks to have size 1 . For the upper packed format, the source-code implementation of PPTRF uses the Level-2 BLAS _TPSV for each column of $U$ to solve the triangular set of equations that determine its off-diagonal entries (see equation (5)). For the lower packed format, PPTRF uses the Level-2 BLAS _SPR [Dongarra et al. 1988] to perform a rank-1 update of the remaining matrix. In both cases, the code is inefficient because the Level-2 BLAS involve an amount of data movement through the cache that is proportional to the number of arithmetic operations performed.

We have written Fortran 90 subroutines _HPPTF and _HPPTS with the same objectives for the blocked hybrid format as _PPTRF and _PPTRS have for the unblocked packed format and their argument lists are very similar. In addition, the subroutines _PPHPP and _HPPPP perform in-place rearrangements between the formats, using the ideas discussed in section 2. These subroutines are available in the companion algorithm [Andersen et al. 2004].

We have used the computers listed in Table 1 with the libraries listed in Table 3 
Table 5. Mflops, Cholesky factorizations including rearrangement, different $n b$ values, IBM Power4.

\begin{tabular}{lccccccccccc}
\hline$n$ & 40 & 64 & 100 & 160 & 250 & 400 & 640 & 1000 & 1600 & 2500 & 4000 \\
\hline Lower Packed Hybrid \\
$n b=40$ & 956 & 1444 & 2055 & 2679 & 3156 & 3626 & 3861 & 3933 & 3968 & 3960 & 4040 \\
$n b=72$ & 957 & 1520 & 1972 & 2650 & 3100 & 3626 & 3971 & 4086 & 4162 & 4166 & 4292 \\
$n b=100$ & 943 & 1494 & 2103 & 2741 & 3193 & 3715 & 3971 & 4119 & 4162 & 4117 & 4258 \\
$n b=200$ & 947 & 1515 & 2116 & 2417 & 2895 & 3440 & 3786 & 4059 & 4213 & 4322 & 4500 \\
\hline
\end{tabular}

Table 6. Size of the packed matrix in megabytes.

\begin{tabular}{lrrrrrrrrrrr}
\hline$n$ & 40 & 64 & 100 & 160 & 250 & 400 & 640 & 1000 & 1600 & 2500 & 4000 \\
Mbytes & 0.006 & 0.016 & 0.039 & 0.098 & 0.239 & 0.612 & 1.6 & 3.8 & 9.8 & 23.9 & 61.1 \\
\hline
\end{tabular}

to compare our blocked hybrid codes with the full-format codes in LAPACK and the recursive packed codes of Andersen, Gustavson, and Waśniewski [2001]. Note that the full-format code does most of its work in calls of _GEMM for subarrays that are not held contiguously in memory and the recursive packed code does most of its work in calls of _GEMM for arrays that are held contiguously but may be too large for level-1 cache. In both cases, the performance is therefore marred by _GEMM doing data copying before starting its actual computation and, possibly, upon completion of that computation.

We compiled the LAPACK source codes with the highest level of optimization available and called the vendor-supplied BLAS. We believe that this is what most users will do, but we also ran the vendor-supplied LAPACK codes or the ATLAS LAPACK codes on the Intel Pentium III where vendor codes were unavailable.

The speeds were obtained by running the code repeatedly until at least a second and a half had elapsed, which gives reasonably reliable figures, though some of the final digits shown in the tables varied when runs were repeated.

For the block size $n b$ in the blocked hybrid codes, we have experimented with the values 40,72, 100, and 200. The first three were used in Section 7.1. We added $n b=200$ to see what would happen with a larger value.

The speeds for factorization, including rearrangement to the packed hybrid format, on the IBM Power4 are shown in Table 5. Since a square matrix of order 72 occupies $41.5 \mathrm{~K}$ bytes of memory, this is the biggest of our $n b$ values to permit full advantage of streaming to be taken in calls of _GEMM. However, this value rarely gives the best speed. For small $n$, the kernel performance is of overriding importance. For $n \geq 640$, the packed matrix does not fit into level- 2 cache, see Table 6 , so a larger value of $n b$ will reduce the movement from level- 3 cache. Our conclusion is that $n b=100$ is suitable for this machine, but note that higher performance is available for large $n$ with $n b=200$.

Similar performance figures for the SUN Ultra III are shown in Table 7. This has a larger level-1 cache, which permits full advantage of streaming to be taken 
Table 7. Mflops, Cholesky factorizations including rearrangement, different $n b$ values, SUN Ultra III.

\begin{tabular}{lrrrrrrrrrrr}
\hline$n$ & 40 & 64 & 100 & 160 & 250 & 400 & 640 & 1000 & 1600 & 2500 & 4000 \\
\hline \multicolumn{2}{l}{ Lower Packed Hybrid } \\
$n b=40$ & 394 & 548 & 644 & 773 & 832 & 963 & 1046 & 1106 & 1110 & 949 & 842 \\
$n b=72$ & 391 & 558 & 660 & 760 & 857 & 993 & 1107 & 1182 & 1215 & 1120 & 1045 \\
$n b=100$ & 389 & 557 & 708 & 738 & 824 & 959 & 1095 & 1115 & 1137 & 1144 & 1083 \\
$n b=200$ & 390 & 557 & 708 & 782 & 867 & 965 & 1080 & 1180 & 1201 & 1206 & 1254 \\
\hline Upper Packed Hybrid & & & & & & & & \\
$n b=40$ & 526 & 608 & 680 & 804 & 830 & 943 & 1006 & 1040 & 1052 & 915 & 791 \\
$n b=72$ & 520 & 769 & 767 & 838 & 916 & 1032 & 1104 & 1182 & 1168 & 1123 & 1004 \\
$n b=100$ & 516 & 770 & 957 & 841 & 901 & 1026 & 1116 & 1177 & 1213 & 1145 & 1065 \\
$n b=200$ & 518 & 768 & 954 & 988 & 983 & 1032 & 1144 & 1181 & 1240 & 1280 & 1243 \\
\hline
\end{tabular}

Table 8. Mflops, Cholesky factorizations including rearrangement, different $n b$ values, SGI Origin 2000 .

\begin{tabular}{lccccccccccc}
\hline$n$ & 40 & 64 & 100 & 160 & 250 & 400 & 640 & 1000 & 1600 & 2500 & 4000 \\
\hline Lower Packed Hybrid & & & & & & & & \\
$n b=40$ & 197 & 199 & 242 & 292 & 339 & 384 & 420 & 445 & 454 & 451 & 447 \\
$n b=72$ & 201 & 269 & 274 & 327 & 366 & 411 & 448 & 430 & 474 & 464 & 450 \\
$n b=100$ & 193 & 269 & 330 & 339 & 382 & 427 & 461 & 484 & 476 & 461 & 439 \\
$n b=200$ & 192 & 264 & 323 & 342 & 359 & 369 & 377 & 397 & 409 & 523 & 529 \\
\hline $\begin{array}{l}\text { Upper Packed Hybrid } \\
n b=40\end{array} 2236$ & 204 & 245 & 290 & 335 & 380 & 416 & 441 & 448 & 442 & 436 \\
$n b=72$ & 237 & 312 & 294 & 331 & 367 & 411 & 447 & 442 & 456 & 459 & 449 \\
$n b=100$ & 235 & 320 & 389 & 352 & 386 & 429 & 462 & 484 & 470 & 458 & 453 \\
$n b=200$ & 237 & 316 & 384 & 381 & 380 & 386 & 383 & 404 & 406 & 488 & 528 \\
\hline
\end{tabular}

in calls of _GEMM when $n b=72$. But, again, the kernel performance is of overriding importance for small $n$ and level-2 cache is important for large $n$ (the packed matrix does not fit in level-2 cache for $n \geq 1600$ ). Here, our conclusion is that $n b=200$ is suitable.

Tables 8 to 11 show the speed variation with $n b$ on our other platforms and they show similar patterns. We conclude that suitable values for $n b$ are 100 for the SGI Origin 2000, 200 for the Alpha EV6, 200 for the Itanium rx2600s and 40 for the Intel Pentium III.

Having chosen $n b$ values, we now make comparisons with other algorithms and show the rearrangement overheads. In Table 12, we show factorization speeds on the IBM Power4 computer. The first two rows show the performance of the LAPACK code _PPTRF for the lower and upper packed formats when compiled with full optimization and calling the vendor-supplied BLAS. Their performance deteriorates markedly as $n$ increases beyond 640 . We believe that this is because they are using Level-2 BLAS. None of the other codes have this defect. It is clear that using Level-2 BLAS is not a good strategy. The ESSL library contains an equivalent code for the lower packed format and we show its speed in the third line of the table. Part of this code is based on the work of Gustavson and Jonsson [2000].

The next four lines show the speeds of comparable codes for the full format and 
Table 9. Mflops, Cholesky factorizations including rearrangement, different $n b$ values, Alpha EV6.

\begin{tabular}{lccccccccccc}
\hline$n$ & 40 & 64 & 100 & 160 & 250 & 400 & 640 & 1000 & 1600 & 2500 & 4000 \\
\hline Lower Packed Hybrid \\
$n b=40$ & 370 & 326 & 380 & 456 & 525 & 576 & 622 & 638 & 645 & 649 & 657 \\
$n b=72$ & 368 & 473 & 396 & 450 & 511 & 582 & 642 & 677 & 694 & 713 & 726 \\
$n b=100$ & 345 & 457 & 497 & 476 & 533 & 600 & 655 & 689 & 712 & 738 & 749 \\
$n b=200$ & 343 & 455 & 491 & 537 & 525 & 591 & 648 & 695 & 731 & 764 & 789 \\
\hline Upper Packed Hybrid & & & & & & & & \\
$n b=40$ & 432 & 347 & 388 & 465 & 518 & 588 & 626 & 645 & 660 & 652 & 655 \\
$n b=72$ & 412 & 531 & 426 & 460 & 521 & 585 & 642 & 659 & 688 & 703 & 711 \\
$n b=100$ & 431 & 525 & 563 & 494 & 538 & 600 & 655 & 689 & 718 & 733 & 742 \\
$n b=200$ & 432 & 521 & 554 & 595 & 566 & 591 & 662 & 701 & 744 & 767 & 790 \\
\hline
\end{tabular}

Table 10. Mflops, Cholesky factorizations including rearrangement, different $n b$ values, Itanium rx2600s.

\begin{tabular}{lrrrrrrrrrrr}
\hline$n$ & 40 & 64 & 100 & 160 & 250 & 400 & 640 & 1000 & 1600 & 2500 & 4000 \\
\hline Lower Packed Hybrid \\
$n b=40$ & 696 & 388 & 510 & 608 & 805 & 1094 & 1418 & 1694 & 1978 & 2225 & 2326 \\
$n b=72$ & 700 & 1034 & 600 & 683 & 827 & 1109 & 1385 & 1687 & 2027 & 2244 & 2449 \\
$n b=100$ & 668 & 1011 & 1701 & 763 & 869 & 1108 & 1419 & 1709 & 2017 & 2284 & 2483 \\
$n b=200$ & 657 & 1017 & 1689 & 1731 & 1279 & 1384 & 1552 & 1829 & 2089 & 2216 & 2402 \\
\hline Upper Packed Hybrid & & & & & & & & \\
$n b=40$ & 805 & 437 & 508 & 674 & 854 & 1144 & 1437 & 1687 & 1941 & 2188 & 2238 \\
$n b=72$ & 800 & 1142 & 680 & 742 & 899 & 1150 & 1419 & 1709 & 2017 & 2274 & 2443 \\
$n b=100$ & 762 & 1107 & 1630 & 831 & 945 & 1180 & 1446 & 1709 & 1998 & 2274 & 2446 \\
$n b=200$ & 768 & 1110 & 1837 & 1805 & 1385 & 1469 & 1609 & 1840 & 2167 & 2468 & 2690 \\
\hline
\end{tabular}

Table 11. Mflops, Cholesky factorizations including rearrangement, different $n b$ values, Intel Pentium III.

\begin{tabular}{lccccccccccc}
\hline$n$ & 40 & 64 & 100 & 160 & 250 & 400 & 640 & 1000 & 1600 & 2500 & 4000 \\
\hline Lower Packed Hybrid \\
$n b=40$ & 116 & 113 & 139 & 182 & 215 & 241 & 261 & 273 & 282 & 284 & 286 \\
$n b=72$ & 118 & 116 & 136 & 169 & 194 & 226 & 257 & 277 & 296 & 308 & 317 \\
$n b=100$ & 111 & 113 & 131 & 169 & 191 & 224 & 244 & 274 & 294 & 307 & 317 \\
$n b=200$ & 108 & 113 & 136 & 137 & 148 & 180 & 219 & 257 & 292 & 310 & 324 \\
\hline $\begin{array}{l}\text { Upper Packed Hybrid } \\
n b=40\end{array} 1131$ & 136 & 157 & 199 & 233 & 259 & 278 & 294 & 300 & 302 & 303 \\
$n b=72$ & 131 & 136 & 150 & 179 & 202 & 226 & 249 & 267 & 280 & 290 & 296 \\
$n b=100$ & 127 & 133 & 148 & 182 & 202 & 227 & 241 & 267 & 282 & 289 & 297 \\
$n b=200$ & 122 & 131 & 152 & 152 & 166 & 199 & 236 & 265 & 288 & 301 & 311 \\
\hline
\end{tabular}


Table 12. Mflops, Cholesky factorizations, $n b=100$, IBM Power4.

\begin{tabular}{lrrrrrrrrrrr}
\hline$n$ & 40 & 64 & 100 & 160 & 250 & 400 & 640 & 1000 & 1600 & 2500 & 4000 \\
Packed LAPACK L & 747 & 951 & 1043 & 1024 & 1059 & 1101 & 1037 & 709 & 638 & 621 & 635 \\
Packed LAPACK U & 530 & 864 & 1201 & 1530 & 1772 & 2038 & 2055 & 1616 & 1460 & 1400 & 1426 \\
Vendor Packed LAPACK L & 1750 & 2359 & 2658 & 2346 & 3107 & 3560 & 3773 & 3870 & 3969 & 3815 & 3836 \\
\hline Full LAPACK L & 440 & 722 & 1390 & 2119 & 2562 & 3242 & 3495 & 3797 & 3901 & 3787 & 4010 \\
Full LAPACK U & 436 & 646 & 1271 & 2063 & 2573 & 3390 & 3810 & 4008 & 4039 & 3975 & 4102 \\
Vendor Full LAPACK L & 1492 & 2165 & 2486 & 3194 & 3454 & 3677 & 3832 & 3921 & 4162 & 4037 & 4327 \\
Vendor Full LAPACK U & 1651 & 2448 & 3020 & 3269 & 3553 & 3878 & 3924 & 4000 & 4137 & 4053 & 4301 \\
\hline Packed Recursive+ L & 170 & 379 & 593 & 1024 & 1586 & 2077 & 2621 & 3030 & 3434 & 3555 & 3943 \\
Packed Recursive L & 181 & 406 & 618 & 1060 & 1652 & 2133 & 2700 & 3111 & 3523 & 3604 & 3980 \\
Packed Recursive+ U & 194 & 418 & 629 & 1122 & 1724 & 2189 & 2931 & 3289 & 3690 & 3801 & 4094 \\
Packed Recursive U & 210 & 444 & 660 & 1185 & 1783 & 2249 & 3031 & 3401 & 3750 & 3858 & 4142 \\
\hline Packed Hybrid+ L & 878 & 1488 & 2085 & 2721 & 3211 & 3754 & 3974 & 4112 & 4188 & 4200 & 4275 \\
Packed Hybrid L & 1006 & 1717 & 2334 & 2977 & 3441 & 3938 & 4149 & 4279 & 4266 & 4269 & 4309 \\
Packed Hybrid+ U & 1090 & 1702 & 2339 & 2792 & 3211 & 3690 & 3832 & 4034 & 4137 & 4150 & 4207 \\
Packed Hybrid U & 1095 & 1702 & 2339 & 2990 & 3416 & 3868 & 4045 & 4194 & 4214 & 4200 & 4249 \\
\hline
\end{tabular}

provide our benchmark. We aim to get similar performance while saving storage with a packed format. We note that the vendor codes are much faster for small $n$, which is probably because the LAPACK code uses Level-2 BLAS (_POTF2) to factorize the blocks on the diagonal, but only slightly faster for $n \geq 1000$ where the speed of _GEMM is of prime importance.

There are two rows for each of the recursive and hybrid formats, according to whether the overheads of rearrangement to this format are included. Whether rearrangment will be needed in practice will vary from case to case. Where the data is generated by computer code, it may be equally efficient to generate it in the chosen format. We do not include rearrangement of the factor back to ordinary packed format since the recursive or hybid format is more suitable for forward and back substitution.

The two lines in Table 12 labelled 'Packed Hybrid+ L' and 'Packed Hybrid+ U' do not show exactly the same times as the rows labelled ' $n b=100$ ' in Table 5 because they come from separate runs, but the differences are very small.

The recursive algorithms achieve performance that approaches that of the LAPACK full codes when the order is large. This is because both are then doing most of their work in significant calls of the Level-3 BLAS _GEMM. However, for smaller $n$, their performance is poor, probably because of the larger ratio of procedure calls to actual computation.

The hybrid algorithm is much faster than the recursive algorithm for small $n$, significantly faster for medium $n$, and slightly faster for large $n$.

If we compare the lower-packed hybrid code with the compiled lower-full LAPACK code, we see that it is always faster, and significantly so for small $n$. We see this as very encouraging. Furthermore, it is slightly faster than the vendor packed code except for small $n$. It is sometimes faster than the vendor full code and would have been faster for all $n \geq 1600$ if we had switched to $n b=200$ at $n=1600$ (see Table 5). The hybrid code for the upper-packed format does not permit us to combine calls of _GEMM, so we expect it not to perform quite so well as the lower-packed 
Table 13. Percentage overheads for rearrangment, IBM Power4.

\begin{tabular}{lrrrrrrrrrrr}
\hline$n$ & 40 & 64 & 100 & 160 & 250 & 400 & 640 & 1000 & 1600 & 2500 & 4000 \\
Packed Recursive L & 6.1 & 6.7 & 4.0 & 3.4 & 4.0 & 2.6 & 2.9 & 2.6 & 2.5 & 1.4 & 0.9 \\
Packed Recursive U & 7.6 & 5.9 & 4.7 & 5.3 & 3.3 & 2.7 & 3.3 & 3.3 & 1.6 & 1.5 & 1.2 \\
Packed Hybrid L & 12.7 & 13.3 & 10.7 & 8.6 & 6.7 & 4.7 & 4.2 & 3.9 & 1.8 & 1.6 & 0.8 \\
Packed Hybrid U & 0.5 & 0.0 & 0.0 & 6.6 & 6.0 & 4.6 & 5.3 & 3.8 & 1.8 & 1.2 & 1.0 \\
\hline
\end{tabular}

Table 14. Mflops, Cholesky Factorizations, $n b=200$, SUN UltraSPARC III.

\begin{tabular}{|c|c|c|c|c|c|c|c|c|c|c|c|}
\hline$n$ & 40 & 64 & 100 & 160 & 250 & 400 & 640 & 1000 & 1600 & 2500 & 4000 \\
\hline Packed LAPACK L & 183 & 247 & 296 & 312 & 321 & 325 & 328 & 331 & 315 & 200 & 169 \\
\hline Packed LAPACK U & 203 & 256 & 302 & 336 & 365 & 393 & 412 & 425 & 409 & 256 & 218 \\
\hline Full LAPACK L & 299 & 436 & 637 & 842 & 933 & 1086 & 864 & 1173 & 1203 & 1169 & 1236 \\
\hline Full LAPACK U & 302 & 403 & 627 & 810 & 942 & 1087 & 1137 & 1211 & 1259 & 1209 & 1050 \\
\hline Packed Recursive+ L & 95 & 202 & 275 & 426 & 550 & 727 & 913 & 1010 & 1093 & 1118 & 1215 \\
\hline Packed Recursive L & 102 & 219 & 290 & 454 & 581 & 760 & 945 & 1043 & 1146 & 1162 & 1249 \\
\hline Packed Recursive+ $\mathrm{U}$ & 90 & 187 & 251 & 381 & 554 & 687 & 864 & 1044 & 1119 & 1173 & 1238 \\
\hline Packed Recursive U & 97 & 201 & 264 & 403 & 586 & 717 & 893 & 1076 & 1176 & 1227 & 1278 \\
\hline Packed Hybrid+ L & 390 & 557 & $\overline{708}$ & 782 & 867 & 965 & 1080 & 1180 & 1201 & 1206 & 1254 \\
\hline Packed Hybrid L & 529 & 778 & 959 & 973 & 1003 & 1077 & 1164 & 1267 & 1277 & 1257 & 1304 \\
\hline Packed Hybrid $+\mathrm{U}$ & 518 & 768 & 954 & 988 & 983 & 1032 & 1144 & 1181 & 1240 & 1280 & 1243 \\
\hline Packed Hybrid U & 520 & 770 & 955 & 988 & 1008 & 1068 & 1178 & 1209 & 1268 & 1280 & 1275 \\
\hline
\end{tabular}

Notes: Vendor Packed Lapack results very similar to Packed Lapack results. Vendor Full Lapack results similar to Full Lapack results.

format, but the difference is slight and it is faster for small $n$ (see next paragraph).

The rearrangement overheads are shown as percentages in Table 13. We see that it is tiny for large $n$, but can be quite significant for small $n$. The higher percentage overhead for the hybrid code is a consequence of the greater speed of the hybrid code and not an inherent inefficiency of the rearrangement. The very small overhead for the upper-packed hybrid case for small $n$ deserve an explanation. This because the upper packed and upper blocked hybrid formats are identical if there is only one block, that is, if $n \leq n b$. Thus the overheads arise only from the procedure call and some simple tests. On the other hand, for the lower blocked hybrid format (Figure 1), the block has to be rearranged from being held by columns to being held by rows.

We show in Table 14, factorization speeds on the SUN UltraSPARC III in the same format as that of Table 12. Here, we do not show the vendor LAPACK speeds since they were very similar to the corresponding speeds for the compiled codes. Once again, the packed LAPACK codes perform poorly, the recursive codes are competitive with the full LAPACK codes for large $n$ but not for small $n$ and the packed hybrid codes significantly outperform the packed recursive codes for small $n$. The hybrid performance is slightly better than that of the recursive code for large $n$, except in one case where the difference is within the timing uncertainty. When we compare the hybrid code with the full LAPACK code, we see that it faster in more than half the cases, with some particularly marked improvements for small $n$.

The rearrangement overheads for our principal packed hybrid code (hybrid L) are less than on the IBM, see Table 15. For the other packed hybrid U code, the 
Table 15. Percentage overheads for rearrangment, SUN UltraSPARC III.

\begin{tabular}{lrrrrrrrrrrr}
\hline$n$ & 40 & 64 & 100 & 160 & 250 & 400 & 640 & 1000 & 1600 & 2500 & 4000 \\
Packed Recursive L & 6.9 & 7.8 & 5.2 & 6.2 & 5.3 & 4.3 & 3.4 & 3.2 & 4.6 & 3.8 & 2.7 \\
Packed Recursive U & 7.2 & 7.0 & 4.9 & 5.5 & 5.5 & 4.2 & 3.2 & 3.0 & 4.8 & 4.4 & 3.1 \\
Packed Hybrid L & 26.3 & 28.4 & 26.2 & 19.6 & 13.6 & 10.4 & 7.2 & 6.9 & 6.0 & 4.1 & 3.8 \\
Packed Hybrid U & 0.4 & 0.3 & 0.1 & 0.0 & 2.5 & 3.4 & 2.9 & 2.3 & 2.2 & 0.0 & 2.5 \\
\hline
\end{tabular}

overheads are similar to those of the packed hybrid L code except when $n$ is small. The rearrangement overheads for the recursive algorithms are broadly comparable with those on the IBM.

We show in Tables 16, 17, 18 and 19, corresponding factorization speeds on our other computers. We do not show the vendor LAPACK speeds since they were always very similar to the corresponding speeds for the compiled codes. The packed LAPACK codes always perform poorly, the recursive codes are always competitive with the full LAPACK codes for large $n$ but not for small $n$ and the packed hybrid codes outperform the packed recursive codes for small $n$. There is a slight fall in the hybrid performance for large $n$ on the Sgi Origin, but not on the other platforms. When we compare the hybrid code with the full LAPACK code, we see that it faster on each machine in more than half the cases, with some particularly marked improvements for small $n$. The performance of the upper hybrid code is on the whole better than that of the lower hybrid code, which is contrary to our expectation.

We do not show separate tables for the rearrangement overheads because they are similar to those on the IBM (Table 13).

Table 16. $\quad$ Mflops, Cholesky Factorizations, $n b=100$, Sgi Origin 2000, R12000.

\begin{tabular}{lrrrrrrrrrrr}
\multicolumn{1}{c}{} & 40 & 64 & 100 & 160 & 250 & 400 & 640 & 1000 & 1600 & 2500 & 4000 \\
\hline Packed LAPACK L & 111 & 144 & 157 & 143 & 144 & 145 & 145 & 143 & 80 & 55 & 35 \\
Packed LAPACK U & 77 & 123 & 167 & 203 & 230 & 251 & 265 & 260 & 121 & 52 & 21 \\
\hline Full LAPACK L & 126 & 207 & 294 & 373 & 446 & 478 & 446 & 444 & 414 & 404 & 331 \\
Full LAPACK U & 116 & 175 & 238 & 306 & 375 & 435 & 474 & 491 & 496 & 446 & 471 \\
\hline Packed Recursive+ L & 38 & 77 & 135 & 225 & 324 & 412 & 462 & 491 & 466 & 453 & 425 \\
Packed Recursive L & 41 & 83 & 145 & 241 & 344 & 431 & 478 & 506 & 484 & 468 & 435 \\
Packed Recursive+ U & 34 & 70 & 119 & 198 & 287 & 370 & 422 & 479 & 465 & 466 & 476 \\
Packed Recursive U & 37 & 74 & 128 & 210 & 302 & 385 & 435 & 493 & 482 & 482 & 489 \\
\hline Packed Hybrid+ L & 193 & 269 & 330 & 339 & 382 & 427 & 461 & 484 & 476 & 461 & 439 \\
Packed Hybrid L & 236 & 324 & 390 & 375 & 411 & 449 & 480 & 499 & 496 & 474 & 447 \\
Packed Hybrid+ U & 235 & 320 & 389 & 352 & 386 & 429 & 462 & 484 & 470 & 458 & 453 \\
Packed Hybrid U & 238 & 322 & 389 & 375 & 411 & 449 & 480 & 500 & 490 & 472 & 460 \\
\hline
\end{tabular}

\subsection{Solution with many right-hand sides}

We next consider the solution for many right-hand sides of a system whose matrix is already factorized. In Table 20, we show the speed on the IBM Power4 for $\max (100, n / 10)$ right-hand sides. There is no rearrangement of the matrix here, since we assume that its factorization has been retained in its recursive or hybrid packed form. For all our values of $n$, the LAPACK codes using the packed formats 
Table 17. Mflops, Cholesky Factorizations, $n b=200$, HP Alpha EV6.

\begin{tabular}{lrrrrrrrrrrr}
\hline$n$ & 40 & 64 & 100 & 160 & 250 & 400 & 640 & 1000 & 1600 & 2500 & 4000 \\
Packed LAPACK L & 198 & 238 & 265 & 276 & 268 & 275 & 273 & 219 & 141 & 134 & 123 \\
Packed LAPACK U & 163 & 238 & 292 & 352 & 374 & 400 & 415 & 325 & 203 & 181 & 184 \\
\hline Full LAPACK L & 318 & 442 & 398 & 469 & 526 & 607 & 607 & 625 & 655 & 679 & 706 \\
Full LAPACK U & 300 & 402 & 411 & 481 & 552 & 619 & 661 & 701 & 724 & 738 & 752 \\
\hline Packed Recursive+ L & 109 & 195 & 267 & 379 & 480 & 535 & 622 & 659 & 666 & 683 & 723 \\
Packed Recursive L & 122 & 216 & 286 & 405 & 508 & 555 & 642 & 674 & 677 & 691 & 729 \\
Packed Recursive+ U & 100 & 186 & 246 & 351 & 458 & 514 & 571 & 618 & 650 & 680 & 733 \\
Packed Recursive U & 111 & 202 & 264 & 376 & 484 & 538 & 588 & 645 & 666 & 691 & 740 \\
\hline Packed Hybrid+ L & 343 & 455 & 491 & 537 & 525 & 591 & 648 & 695 & 731 & 764 & 789 \\
Packed Hybrid L & 426 & 498 & 566 & 596 & 562 & 618 & 669 & 720 & 751 & 779 & 799 \\
Packed Hybrid+ U & 432 & 521 & 554 & 595 & 566 & 591 & 662 & 701 & 744 & 767 & 790 \\
Packed Hybrid U & 437 & 521 & 554 & 595 & 572 & 616 & 669 & 714 & 758 & 781 & 799 \\
\hline
\end{tabular}

Table 18. Mflops, Cholesky Factorizations, $n b=200$, HP-UX Itanium rx2600s.

\begin{tabular}{lrrrrrrrrrrr}
\hline$n$ & 40 & 64 & 100 & 160 & 250 & 400 & 640 & 1000 & 1600 & 2500 & 4000 \\
Packed LAPACK L & 218 & 328 & 463 & 644 & 825 & 952 & 1039 & 660 & 609 & 595 & 590 \\
Packed LAPACK U & 167 & 224 & 272 & 320 & 359 & 387 & 406 & 416 & 425 & 430 & 433 \\
\hline Full LAPACK L & 376 & 581 & 838 & 1307 & 1728 & 2189 & 2546 & 2777 & 2904 & 3028 & 3141 \\
Full LAPACK U & 433 & 734 & 559 & 633 & 889 & 1146 & 1385 & 1821 & 2068 & 2540 & 2676 \\
\hline Packed Recursive+ L & 110 & 232 & 378 & 698 & 1079 & 1608 & 2170 & 2531 & 2758 & 2861 & 3013 \\
Packed Recursive L & 121 & 256 & 411 & 742 & 1147 & 1675 & 2305 & 2666 & 2874 & 2942 & 3056 \\
Packed Recursive+ U & 82 & 176 & 313 & 580 & 951 & 1479 & 1967 & 2424 & 2576 & 2726 & 2867 \\
Packed Recursive U & 89 & 187 & 333 & 616 & 1004 & 1551 & 2063 & 2531 & 2659 & 2785 & 2910 \\
\hline Packed Hybrid+ L & 657 & 1017 & 1689 & 1731 & 1279 & 1384 & 1552 & 1829 & 2089 & 2216 & 2402 \\
Packed Hybrid L & 763 & 1139 & 1849 & 1815 & 1390 & 1504 & 1661 & 1910 & 2167 & 2274 & 2438 \\
Packed Hybrid+ U & 768 & 1110 & 1837 & 1805 & 1385 & 1469 & 1609 & 1840 & 2167 & 2468 & 2690 \\
Packed Hybrid U & 768 & 1124 & 1849 & 1805 & 1394 & 1489 & 1664 & 1886 & 2214 & 2480 & 2724 \\
\hline
\end{tabular}

Table 19. Mflops, Cholesky Factorizations, $n b=40$, Intel Pentium III.

\begin{tabular}{lrrrrrrrrrrr}
\hline$n$ & 40 & 64 & 100 & 160 & 250 & 400 & 640 & 1000 & 1600 & 2500 & 4000 \\
Packed LAPACK L & 111 & 130 & 104 & 99 & 95 & 57 & 41 & 37 & 36 & 35 & 35 \\
Packed LAPACK U & 96 & 127 & 130 & 137 & 146 & 102 & 82 & 77 & 75 & 72 & 71 \\
\hline Full LAPACK L & 106 & 113 & 161 & 193 & 226 & 247 & 266 & 289 & 300 & 298 & 297 \\
Full LAPACK U & 81 & 98 & 144 & 180 & 210 & 237 & 213 & 278 & 244 & 303 & 325 \\
\hline Packed Recursive+ L & 32 & 60 & 93 & 148 & 161 & 199 & 225 & 259 & 288 & 306 & 325 \\
Packed Recursive L & 34 & 64 & 101 & 160 & 170 & 212 & 236 & 271 & 296 & 313 & 330 \\
Packed Recursive+ U & 34 & 66 & 101 & 161 & 175 & 210 & 233 & 263 & 288 & 307 & 325 \\
Packed Recursive U & 36 & 72 & 111 & 175 & 187 & 223 & 242 & 273 & 296 & 313 & 330 \\
\hline Packed Hybrid+ L & 117 & 113 & 139 & 183 & 215 & 241 & 261 & 276 & 284 & 285 & 287 \\
Packed Hybrid L & 134 & 131 & 156 & 202 & 236 & 258 & 274 & 288 & 292 & 290 & 290 \\
Packed Hybrid+ U & 132 & 135 & 156 & 201 & 232 & 263 & 281 & 294 & 303 & 304 & 305 \\
Packed Hybrid U & 134 & 151 & 174 & 222 & 253 & 283 & 295 & 307 & 311 & 309 & 308 \\
\hline
\end{tabular}

perform less well than the other codes. The hybrid codes give performance that is always at least as good as the recursive codes, and is much better for small $n$.

We show comparable figures for the other systems in Tables 21, 22, 23, 24, and 25. We see that the performance of the packed LAPACK codes is poor in 
Table 20. Mflops, Solution, many right-hand sides, Notes: Results for Vendor Packed Lapack L and Vendor Full Lapack very similar to corresponding Lapack results. $n b=100, m b=100$, IBM Power4.

\begin{tabular}{lrrrrrrrrrrr}
\hline$n$ & 40 & 64 & 100 & 160 & 250 & 400 & 640 & 1000 & 1600 & 2500 & 4000 \\
Packed LAPACK L & 919 & 1298 & 1577 & 1822 & 2027 & 2182 & 2088 & 1733 & 1526 & 1460 & 1456 \\
Packed LAPACK U & 912 & 1296 & 1572 & 1836 & 2044 & 2197 & 2088 & 1710 & 1517 & 1467 & 1469 \\
\hline Full LAPACK L & 3195 & 3368 & 3750 & 4021 & 4210 & 4266 & 4259 & 4257 & 4441 & 4320 & 4368 \\
Full LAPACK U & 3205 & 3392 & 3740 & 4045 & 4175 & 4238 & 4259 & 4222 & 4495 & 4320 & 4353 \\
\hline Packed Recursive L & 1240 & 1510 & 1956 & 2658 & 3075 & 3306 & 3524 & 3734 & 4147 & 4166 & 4571 \\
Packed Recursive U & 1225 & 1493 & 1935 & 2655 & 3091 & 3306 & 3510 & 3750 & 4147 & 4166 & 4555 \\
\hline Packed Hybrid L & 2359 & 2941 & 3411 & 3778 & 3991 & 4084 & 4231 & 4285 & 4362 & 4422 & 4620 \\
Packed Hybrid U & 2374 & 2927 & 3389 & 3736 & 3965 & 4132 & 4231 & 4293 & 4415 & 4380 & 4555 \\
\hline
\end{tabular}

Notes: Results for Vendor Packed Lapack L and Vendor Full Lapack very similar to corresponding Lapack results.

every case and is always inferior to the other codes. The hybrid codes always give performance that is reasonally close to that of the full code. It is slightly inferior for small $n$ but slightly superior for large $n$. On the SUN, Sgi Origin, and HP Alpha, the recursive codes are inferior to the hybrid codes for small $n$, but superior for large $n$; this is particularly marked on the SUN. For the Itanium, the recursive code is remarkably successful, consistently out-performing the full code and the hybrid code. On the Pentium III, the full, recursive, and hybrid codes have broadly comparable performance.

We are very pleased to see that the performance of the hybrid code continues to improve as $n$ gets very large, which demonstrates the success of working with blocks of $m b$ columns and rearranging each to the form illustrated in Figure 7. We believe that this explains why the packed hybrid codes outperform the Full LAPACK codes for large $n$ on the SUN, Origin, Alpha, and Itanium. For $n \leq n b$, the rearrangement probably does not speed up the solution since the columns of $B$ are already contiguous in memory, but we have not written special code that avoids the rearrangement in this case.

Table 21. Mflops, Solution, many right-hand sides, $n b=200, m b=100$, SUN UltraSPARC III.

\begin{tabular}{lrrrrrrrrrrr}
\hline$n$ & 40 & 64 & 100 & 160 & 250 & 400 & 640 & 1000 & 1600 & 2500 & 4000 \\
Packed LAPACK L & 289 & 341 & 373 & 265 & 220 & 209 & 203 & 199 & 119 & 63 & 54 \\
Packed LAPACK U & 276 & 326 & 357 & 294 & 271 & 277 & 281 & 270 & 179 & 99 & 87 \\
\hline Full LAPACK L & 901 & 933 & 1045 & 1089 & 1103 & 1279 & 1238 & 1300 & 1268 & 1312 & 1299 \\
Full LAPACK U & 894 & 974 & 1042 & 1095 & 1103 & 1276 & 1244 & 1312 & 1261 & 1390 & 1290 \\
\hline Packed Recursive L & 280 & 467 & 489 & 678 & 849 & 981 & 1081 & 1241 & 1296 & 1362 & 1436 \\
Packed Recursive U & 267 & 466 & 477 & 659 & 843 & 968 & 1071 & 1239 & 1291 & 1360 & 1434 \\
\hline Packed Hybrid L & 766 & 852 & 959 & 1023 & 1083 & 1236 & 1317 & 1334 & 1373 & 1420 & 1436 \\
Packed Hybrid U & 767 & 858 & 959 & 1031 & 1074 & 1234 & 1316 & 1336 & 1401 & 1453 & 1435 \\
\hline
\end{tabular}

Notes: Vendor Packed Lapack results very similar to Packed Lapack results (slightly inferior for small $n$ ). Vendor Full Lapack results inferior to Full Lapack results. 
Table 22. Mflops, Solution, many right-hand sides, $n b=100, m b=100$, Sgi Origin 2000, R12000.

\begin{tabular}{lrrrrrrrrrrr}
\hline$n$ & 40 & 64 & 100 & 160 & 250 & 400 & 640 & 1000 & 1600 & 2500 & 4000 \\
Packed LAPACK L & 125 & 162 & 185 & 201 & 212 & 218 & 221 & 170 & 57 & 56 & 39 \\
Packed LAPACK U & 123 & 161 & 184 & 200 & 211 & 218 & 220 & 171 & 51 & 53 & 38 \\
\hline Full LAPACK L & 264 & 330 & 394 & 442 & 483 & 510 & 510 & 507 & 492 & 481 & 447 \\
Full LAPACK U & 260 & 316 & 373 & 420 & 461 & 496 & 491 & 512 & 477 & 476 & 446 \\
\hline Packed Recursive L & 206 & 209 & 319 & 370 & 434 & 475 & 483 & 516 & 497 & 480 & 484 \\
Packed Recursive U & 206 & 209 & 319 & 370 & 434 & 475 & 487 & 516 & 498 & 494 & 487 \\
\hline Packed Hybrid L & 229 & 290 & 345 & 403 & 444 & 482 & 504 & 509 & 513 & 517 & 510 \\
Packed Hybrid U & 228 & 290 & 345 & 402 & 445 & 481 & 504 & 509 & 503 & 518 & 506 \\
\hline
\end{tabular}

Notes: Results for Vendor Packed Lapack and Vendor Full Lapack very similar to corresponding Lapack results.

\begin{tabular}{lrrrrrrrrrrr} 
Table 23. Mflops, Solution, many right-hand sides, $n b=200, m b=100$, HP Alpha EV6 \\
\hline$n$ & 40 & 64 & 100 & 160 & 250 & 400 & 640 & 1000 & 1600 & 2500 & 4000 \\
Packed LAPACK L & 224 & 293 & 360 & 380 & 403 & 421 & 427 & 318 & 254 & 203 & 203 \\
Packed LAPACK U & 226 & 296 & 358 & 384 & 403 & 428 & 422 & 330 & 219 & 203 & 197 \\
\hline Full LAPACK L & 443 & 478 & 556 & 624 & 669 & 718 & 732 & 712 & 750 & 759 & 762 \\
Full LAPACK U & 444 & 488 & 553 & 625 & 668 & 709 & 732 & 727 & 750 & 756 & 761 \\
\hline Packed Recursive L & 328 & 395 & 425 & 576 & 649 & 702 & 687 & 712 & 687 & 732 & 766 \\
Packed Recursive U & 324 & 389 & 432 & 579 & 635 & 702 & 672 & 712 & 687 & 732 & 766 \\
\hline Packed Hybrid L & 407 & 460 & 526 & 597 & 656 & 722 & 739 & 774 & 792 & 804 & 827 \\
Packed Hybrid U & 413 & 448 & 522 & 597 & 645 & 717 & 747 & 774 & 799 & 811 & 823 \\
\hline
\end{tabular}

Notes: Results for Vendor Packed Lapack same as Packed Lapack and for Vendor Full Lapack same as Full Lapack, apart from timing uncertainties.

\begin{tabular}{lrrrrrrrrrrrr} 
Table 24. Mflops, Solution, many right-hand sides, $n b=200, m b=100$, HP-UX Itanium rx2600s. \\
\hline & 40 & 64 & 100 & 160 & 250 & 400 & 640 & 1000 & 1600 & 2500 & 4000 \\
Packed LAPACK L & 223 & 305 & 385 & 471 & 538 & 576 & 608 & 628 & 640 & 649 & 655 \\
Packed LAPACK U & 225 & 308 & 385 & 465 & 532 & 578 & 614 & 632 & 642 & 648 & 655 \\
\hline Full LAPACK L & 272 & 411 & 593 & 844 & 1150 & 1525 & 1878 & 2222 & 2452 & 2637 & 2813 \\
Full LAPACK U & 271 & 406 & 593 & 835 & 1157 & 1538 & 1881 & 2222 & 2452 & 2626 & 2813 \\
\hline Packed Recursive L & 712 & 958 & 1325 & 1641 & 2071 & 2440 & 2678 & 2765 & 3015 & 3094 & 3062 \\
Packed Recursive U & 695 & 958 & 1289 & 1617 & 2077 & 2392 & 2636 & 2784 & 3015 & 3094 & 3062 \\
\hline Packed Hybrid L & 251 & 396 & 581 & 817 & 1103 & 1473 & 1853 & 2196 & 2560 & 2765 & 2976 \\
Packed Hybrid U & 251 & 396 & 577 & 808 & 1103 & 1463 & 1853 & 2193 & 2544 & 2777 & 2969 \\
\hline
\end{tabular}

Notes: Results for Vendor Packed Lapack same as Packed Lapack and for Vendor Full Lapack same as Full Lapack, apart from timing uncertainties. No significant difference with $m b=200$ or $m b=48$.

\subsection{Solution with one right-hand side}

Finally, we have measured the speeds while solving for single right-hand side, for a system whose matrix is already factorized. The results are shown in Tables 26 to 31. It is inherently more difficult to obtain a high speed when $n$ is large since the matrix will need to be read into cache once for forward substitution and once for back-substitution. It should therefore be possible for the packed codes to execute faster than the full codes. For the LAPACK codes, this is quite often the case; it is particularly so for large $n$ on the Sgi Origin and the Itanium. However, on the SUN, the packed LAPACK codes do not perform well. 
Table 25. Mflops, Solution, many right-hand sides, $n b=40, m b=100$, Intel Pentium III.

\begin{tabular}{lrrrrrrrrrrr}
\hline$n$ & 40 & 64 & 100 & 160 & 250 & 400 & 640 & 1000 & 1600 & 2500 & 4000 \\
Packed LAPACK L & 110 & 128 & 123 & 120 & 123 & 94 & 67 & 60 & 55 & 54 & 53 \\
Packed LAPACK U & 114 & 132 & 127 & 123 & 124 & 95 & 67 & 59 & 55 & 53 & 53 \\
\hline Full LAPACK L & 160 & 131 & 223 & 259 & 277 & 300 & 262 & 317 & 339 & 346 & 327 \\
Full LAPACK U & 161 & 192 & 231 & 259 & 274 & 294 & 262 & 319 & 341 & 346 & 325 \\
\hline Packed Recursive L & 164 & 160 & 230 & 228 & 274 & 290 & 267 & 314 & 330 & 342 & 352 \\
Packed Recursive U & 163 & 157 & 225 & 226 & 271 & 285 & 268 & 310 & 328 & 341 & 352 \\
\hline Packed Hybrid L & 144 & 187 & 221 & 256 & 277 & 300 & 313 & 314 & 311 & 315 & 317 \\
Packed Hybrid U & 141 & 188 & 222 & 257 & 277 & 302 & 315 & 314 & 305 & 310 & 314 \\
\hline
\end{tabular}

Notes: Results for Vendor Packed Lapack within timing uncertainties of Packed Lapack Results for Vendor Full Lapack same as Full Lapack, apart from timing uncertainties.

Table 26. Mflops, Solution, one right-hand side, $n b=40$, IBM Power4.

\begin{tabular}{lrrrrrrrrrr}
\hline$n$ & 40 & 64 & 100 & 160 & 250 & 400 & 640 & 1000 & 1600 & 2500 \\
Packed LAPACK L & 906 & 1205 & 1496 & 1798 & 2021 & 2253 & 1907 & 1645 & 1479 & 1432 \\
Packed LAPACK U & 899 & 1187 & 1488 & 1814 & 2022 & 2275 & 1868 & 1612 & 1508 & 1473 \\
Vendor Packed Lapack L & 894 & 1196 & 1489 & 1789 & 2022 & 2257 & 1892 & 1592 & 1488 & 1479 \\
\hline Full LAPACK L & 896 & 1175 & 1505 & 1852 & 2073 & 2206 & 1669 & 1331 & 1358 & 1432 \\
Full LAPACK U & 885 & 1178 & 1537 & 1804 & 2085 & 2217 & 1578 & 1294 & 1313 & 1390 \\
Vendor Full LAPACK L & 896 & 1161 & 1512 & 1824 & 2069 & 2204 & 1683 & 1309 & 1356 & 1430 \\
Vendor Full LAPACK U & 860 & 1152 & 1520 & 1793 & 2066 & 2231 & 1579 & 1297 & 1306 & 1444 \\
\hline Packed Recursive L & 283 & 417 & 555 & 810 & 887 & 1335 & 1299 & 1095 & 1239 & 1290 \\
Packed Recursive U & 280 & 419 & 558 & 808 & 907 & 1344 & 1356 & 1107 & 1242 & 1274 \\
\hline Packed Hybrid L & 880 & 1177 & 1498 & 1647 & 1851 & 2098 & 1480 & 1150 & 1027 & 1009 \\
Packed Hybrid U & 874 & 1167 & 1474 & 1622 & 1826 & 1993 & 1452 & 1121 & 1017 & 993 \\
\hline
\end{tabular}

Table 27. Mflops, Solution, one right-hand side, $n b=200$, SUN UltraSPARC III.

\begin{tabular}{lrrrrrrrrrrr}
\hline$n$ & 40 & 64 & 100 & 160 & 250 & 400 & 640 & 1000 & 1600 & 2500 & 4000 \\
Packed LAPACK L & 285 & 339 & 383 & 268 & 221 & 208 & 204 & 199 & 84 & 56 & 51 \\
Packed LAPACK U & 270 & 325 & 371 & 290 & 274 & 280 & 281 & 280 & 154 & 96 & 85 \\
\hline Full LAPACK L & 363 & 479 & 575 & 657 & 665 & 798 & 798 & 495 & 370 & 285 & 286 \\
Full LAPACK U & 373 & 472 & 601 & 698 & 667 & 786 & 791 & 540 & 340 & 306 & 288 \\
\hline Packed Recursive L & 71 & 113 & 155 & 229 & 299 & 404 & 504 & 560 & 318 & 258 & 252 \\
Packed Recursive U & 72 & 114 & 155 & 229 & 306 & 405 & 505 & 566 & 314 & 263 & 277 \\
\hline Packed Hybrid L & 247 & 310 & 354 & 294 & 338 & 411 & 519 & 554 & 348 & 265 & 229 \\
Packed Hybrid U & 240 & 309 & 352 & 292 & 337 & 411 & 520 & 584 & 325 & 231 & 224 \\
\hline
\end{tabular}

Notes: Vendor Packed Lapack results inferior to Full Lapack results.

For small $n$, the hybrid codes give consistently better performance than the recursive codes, and they are broadly comparable with the packed LAPACK codes.

For large $n$, the recursive code is usually better than the hybrid code, but there is a notable exception on the Pentium III (Table 31) and there is little difference on the Sgi Origin (Table 28). When compared to the best LAPACK code, the recursive code is usually slightly slower but is much faster on the Sgi Origin and Itanium. Where the packed LAPACK code performs well, consideration should be given to rearranging the factorized matrix back to the packed format.

For the IBM processor, we have omitted the results for $n=4000$ since those that 


\begin{tabular}{lrrrrrrrrrrr} 
Table 28. & Mflops, Solution, one right-hand side, $n b=100$, Sgi Origin $2000, \mathrm{R} 12000$. \\
\hline$n$ & 40 & 64 & 100 & 160 & 250 & 400 & 640 & 1000 & 1600 & 2500 & 4000 \\
Packed LAPACK L & 117 & 157 & 199 & 208 & 211 & 217 & 215 & 184 & 78 & 59 & 55 \\
Packed LAPACK U & 116 & 156 & 193 & 204 & 209 & 215 & 213 & 182 & 77 & 56 & 56 \\
\hline Full LAPACK L & 166 & 236 & 254 & 258 & 260 & 263 & 161 & 120 & 33 & 19 & 12 \\
Full LAPACK U & 175 & 230 & 252 & 243 & 283 & 270 & 160 & 160 & 34 & 19 & 12 \\
\hline Packed Recursive L & 30 & 45 & 66 & 98 & 133 & 171 & 210 & 214 & 82 & 88 & 74 \\
Packed Recursive U & 30 & 45 & 67 & 100 & 132 & 174 & 215 & 216 & 82 & 87 & 86 \\
\hline Packed Hybrid L & 115 & 151 & 190 & 233 & 270 & 295 & 323 & 327 & 91 & 58 & 87 \\
Packed Hybrid U & 112 & 148 & 187 & 231 & 266 & 291 & 313 & 316 & 83 & 60 & 53 \\
\hline
\end{tabular}

Notes: Results for Vendor Packed Lapack and Vendor Full Lapack very similar to corresponding Lapack results.

Table 29. $\quad$ Mflops, Solution, one right-hand side, $n b=200$, HP Alpha EV6.

\begin{tabular}{lrrrrrrrrrrr}
\hline$n$ & 40 & 64 & 100 & 160 & 250 & 400 & 640 & 1000 & 1600 & 2500 & 4000 \\
Packed LAPACK L & 217 & 290 & 351 & 400 & 409 & 425 & 424 & 322 & 228 & 203 & 192 \\
Packed LAPACK U & 220 & 289 & 358 & 399 & 406 & 423 & 410 & 306 & 238 & 214 & 192 \\
\hline Full LAPACK L & 190 & 249 & 298 & 312 & 324 & 338 & 283 & 257 & 217 & 199 & 192 \\
Full LAPACK U & 191 & 243 & 286 & 304 & 320 & 334 & 289 & 263 & 214 & 197 & 174 \\
\hline Packed Recursive L & 105 & 152 & 200 & 245 & 286 & 312 & 354 & 261 & 194 & 172 & 174 \\
Packed Recursive U & 102 & 152 & 200 & 242 & 280 & 317 & 344 & 257 & 194 & 172 & 174 \\
\hline Packed Hybrid L & 215 & 293 & 345 & 379 & 377 & 403 & 384 & 253 & 201 & 183 & 174 \\
Packed Hybrid U & 218 & 284 & 343 & 382 & 381 & 403 & 379 & 258 & 201 & 183 & 160 \\
\hline
\end{tabular}

Notes: Results for Vendor Packed Lapack within timing uncertainties of Packed Lapack Results for Vendor Full Lapack same as Full Lapack, apart from timing uncertainties.

\begin{tabular}{lrrrrrrrrrrr} 
Table 30. & Mflops, Solution, one right-hand side, $n b=200$, HP-UX Itanium rx2600s. \\
\hline$n$ & 40 & 64 & 100 & 160 & 250 & 400 & 640 & 1000 & 1600 & 2500 & 4000 \\
Packed LAPACK L & 221 & 301 & 382 & 471 & 518 & 587 & 655 & 622 & 640 & 649 & 639 \\
Packed LAPACK U & 225 & 301 & 385 & 462 & 542 & 584 & 738 & 663 & 657 & 653 & 640 \\
\hline Full LAPACK L & 181 & 263 & 341 & 452 & 522 & 625 & 317 & 307 & 314 & 285 & 266 \\
Full LAPACK U & 183 & 261 & 344 & 438 & 554 & 603 & 344 & 312 & 317 & 287 & 266 \\
\hline Packed Recursive L & 76 & 118 & 173 & 268 & 370 & 529 & 615 & 662 & 779 & 886 & 1065 \\
Packed Recursive U & 72 & 118 & 169 & 258 & 382 & 527 & 591 & 658 & 771 & 888 & 799 \\
\hline Packed Hybrid L & 194 & 279 & 371 & 449 & 618 & 847 & 743 & 649 & 624 & 620 & 640 \\
Packed Hybrid U & 193 & 280 & 370 & 450 & 620 & 855 & 776 & 670 & 640 & 633 & 639 \\
\hline
\end{tabular}

Notes: Results for Vendor Packed Lapack within timing uncertainties of Packed Lapack except that Vendor Packed Lapack achieved 799 Mflops for $n=4000$. Results for Vendor Full Lapack same as Full Lapack, apart from timing uncertainties.

we obtained look unreliable. We are investigating this case further.

\section{SUMMARY AND CONCLUSIONS}

Our primary goal when we commenced this work was to investigate how much better the recursive algorithm performed than a simple blocking algorithm. We expected that it to be better since it can take advantage of all levels of cache, but did not know by how much. What we have found is that it tends to be inferior for systems of small order where a single well-chosen block size can take good advantage of level-1 cache, often by a factor of about 3-4 for our implementations. 
Table 31. Mflops, Solution, one right-hand side, $n b=40$, Intel Pentium III.

\begin{tabular}{lrrrrrrrrrrrr}
\hline$n$ & 40 & 64 & 100 & 160 & 250 & 400 & 640 & 1000 & 1600 & 2500 & 4000 \\
Packed LAPACK L & 112 & 121 & 120 & 128 & 100 & 67 & 59 & 57 & 55 & 54 & 54 \\
Packed LAPACK U & 115 & 140 & 131 & 133 & 119 & 85 & 65 & 59 & 54 & 53 & 54 \\
\hline Full LAPACK L & 38 & 48 & 68 & 90 & 75 & 70 & 61 & 56 & 57 & 52 & 45 \\
Full LAPACK U & 39 & 51 & 73 & 95 & 83 & 69 & 60 & 57 & 56 & 52 & 44 \\
\hline Packed Recursive L & 12 & 19 & 28 & 42 & 44 & 51 & 52 & 52 & 52 & 49 & 45 \\
Packed Recursive U & 12 & 19 & 28 & 42 & 44 & 50 & 51 & 51 & 52 & 48 & 46 \\
\hline Packed Hybrid L & 100 & 108 & 121 & 139 & 146 & 120 & 110 & 109 & 109 & 109 & 106 \\
Packed Hybrid U & 91 & 108 & 120 & 137 & 129 & 111 & 101 & 98 & 98 & 97 & 99 \\
\hline
\end{tabular}

Notes: Results for Vendor Packed Lapack within timing uncertainties of Packed Lapack Results for Vendor Full Lapack same as Full Lapack, apart from timing uncertainties.

For large systems, performance of the recursive algorithm is similar on the SUN and Sgi, worse on the IBM, Alpha, and better only on the Itanium and Pentium. An advantage of the recursive method, of course, is that no choice of block size is needed.

On balance, we therefore see that the hybrid algorithm performs better than the recursive algorithm. Also, we see that it often outperforms the full-storage LAPACK code.

In the course of this investigation, we have considered carefully exactly how the blocks should be chosen to permit good advantage to be taken of level-1 cache during factorization and solution to allow rapid rearrangement to the block form without undue use of temporary memory. We noted that where the data is generated by computer code, it may be equally efficient to generate it in the chosen format so that no rearrangement is needed.

We have also developed some new kernel codes for the Cholesky factorization of the diagonal blocks because the LAPACK kernel POTF2 is unsatisfactory as it uses Level-2 BLAS. We obtained remarkably fast performance for our kernel by using mini-blocks of order 2 in standard Fortran code.

\section{ACKNOWLEDGEMENTS}

The work described here is partly the outcome of collaborations with HPCN at the University of Umea, Sweden and UNI•C in Lyngby, Denmark. At Umea we thank Isak Jonsson.

We would like to thank Niels Carl W Hansen for consulting on the IBM and SGI systems; Bernd Dammann for consulting on the SUN system; Susanne Balle and Martin Antony Walker for making the HP Itanium 2 system available to us; Tim Regan for consulting on the HP Itanium 2 system; and Minka and Alexander Karaivanov for several discussions. 


\section{REFERENCES}

Agarwal, R., Gustavson, F., and Zubair, M. 1994. Exploiting functional parallelism on power2 to design high-performance numerical algorithms. IBM Journal of Research and Development 38, 5 (September), 563-576.

Andersen, B., Gunnels, J., Gustavson, F., Reid, J., and Waśniewski, J. $2004 . \quad$ Fortran 90 Subroutines for the Cholesky Algorithm in Blocked Hybrid Format. For submission to the ACM Transactions on Mathematical Software.

Andersen, B., Gunnels, J., Gustavson, F., And Waśniewski, J. 2002. A Recursive Formulation of the Inversion of symmetric positive definite Matrices in Packed Storage Data Format. In J. Fagerholm, J. HaAtaja, J. Järvinen, M. Lyly, and P. R. V. Savolainen Eds., Proceedings of the $6^{\text {th }}$ International Conference, PARA 2002, Applied Parallel Computing, Number 2367 in Lecture Notes in Computer Science (Espoo, Finland, June 2002), pp. 287-296. Springer.

Andersen, B., Gustavson, F., And Waśniewski, J. 2001. A Recursive Formulation of Cholesky Facorization of a Matrix in Packed Storage. ACM Transactions on Mathematical Software 27, 2 (Jun), 214-244.

Anderson, E., Bai, Z., Bischof, C., Blackford, L. S., Demmel, J., Dongarra, J., Du Croz, J., Greenbaum, A., Hammarling, S., McKenney, A., and Sorensen, D. 1999. LAPACK Users' Guide (Third ed.). Society for Industrial and Applied Mathematics, Philadelphia, PA.

Calahan, D. 1986. Block-Oriented local memory-based linear equation solution on the Cray-2; Uniprocessor algorithms. In Proceedings International Conference on Parallel Processing, IEEE Computer Society Press (Yew York, USA, August 1986).

Chatterjee, S., Jain, V. V., Lebeck, A. R., Mundhra, S., and Thottethodi, M. 1999. Nonlinear array layouts for hierarchical memory systems. In International Conference on Supercomputing (1999), pp. 444-453.

Dongarra, J., Bunch, J., Moler, C., And Stewart, G. 1979. Linpack Users' Guide. Society for Industrial and Applied Mathematics, Philadelphia, PA, USA.

Dongarra, J., Du Croz, J., Duff, I. S., and Hammarling, S. 1990. A Set of Level 3 Basic Linear Algebra Subprograms. ACM Trans. Math. Soft. 16, 1 (March), 1-17.

Dongarra, J., Du Croz, J., Hammarling, S., and Hanson, R. J. 1988. An Extended Set of Fortran Basic Linear Algebra Subroutines. ACM Trans. Math. Soft. 14, 1 (March), $1-17$.

DufF, I. S. AND ReID, J. K. 1996. Exploiting zeros on the diagonal in the direct solution of indefinite sparse symmetric linear systems. ACM Transactions on Mathematical Software 22, 2, 227-257.

Frens, J. D. AND WiSE, D. S. 1997. Auto-blocking matrix-multiplication or tracking blas3 performance from source code. In Proceedings of the sixth ACM SIGPLAN symposium on Principles and practice of parallel programming (1997), pp. 206-216. ACM Press.

Gallivan, K., Jalby, W., Meier, U., and Sameh, A. 1987. The impact of hierarchical memory systems on linear algebra algorithm design. CSRD Report 625 (SEP), CSRD.

Gustavson, F. 1997. Recursion Leads to Automatic Variable Blocking for Dense LinearAlgebra Algorithms. IBM Journal of Research and Development 41, 6 (November), 737755.

Gustavson, F. And Jonsson, I. 2000. Minimal storage high performance cholesky via blocking and recursion. IBM Journal of Research and Development 44, 6 (Nov), 823-849.

IBM. 1986. Engineering and Scientific Subroutine Library, Guide and Reference. First Edition (Program Number 5668-863).

Lawson, C. L., Hanson, R. J., Kincaid, D., And Krogh, F. T. 1979. Basic Linear Algebra Subprograms for Fortran Usage. ACM Trans. Math. Soft. 5, 308-323.

Valsalam, V. AND SkJEllum, A. 2002. A framework for high-performance matrix multiplication based on hierarchical abstractions, algorithms and optimized low-level kernels. 14, 10 (Aug.), 805-839. 
Waśniewski, J., Andersen, B., And Gustavson, F. 1998. Recursive Formulation of Cholesky Algorithm in Fortran 90. In B. KÅgström, J. Dongarra, E. Elmroth, And J. WaŚniewski Eds., Proceedings of the $4^{\text {th }}$ International Workshop, Applied Parallel Computing, Large Scale Scientific and Industrial Problems, PARA'98, Number 1541 in Lecture Notes in Computer Science Number (Umeå, Sweden, June 1998), pp. 574-578. Springer.

Whaley, R., Petitet, A., And Dongarra, J. 2000. ATlAS: Automatically Tuned Linear Algebra Software. http://www.netlib.org/atlas/. University of Tennessee at Knoxville, Tennessee, USA. 